\title{
Constraints on the Cosmic-Ray Density Gradient beyond the Solar Circle from Fermi $\gamma$-ray Observations of the Third Galactic Quadrant
}

\author{
M. Ackermann ${ }^{2}$, M. Ajello ${ }^{2}$, L. Baldini ${ }^{3}$, J. Ballet ${ }^{4}$, G. Barbiellini ${ }^{5,6}$, D. Bastieri ${ }^{7,8}$, K. Bechtol ${ }^{2}$, R. Bellazzini $^{3}$, \\ B. Berenji ${ }^{2}$, E. D. Bloom ${ }^{2}$, E. Bonamente ${ }^{9,10}$, A. W. Borgland ${ }^{2}$, T. J. Brandt ${ }^{11,12}$, J. Bregeon ${ }^{3}$, A. Brez $^{3}$, \\ M. Brigida ${ }^{13,14}$, P. Bruel ${ }^{15}$, R. Buehler ${ }^{2}$, S. Buson ${ }^{7,8}$, G. A. Caliandro ${ }^{16}$, R. A. Cameron ${ }^{2}$, P. A. Caraveo ${ }^{17}$, \\ J. M. Casandjian ${ }^{4}$, C. Cecchi ${ }^{9,10}$, E. Charles ${ }^{2}$, A. Chekhtman ${ }^{18,19}$, J. Chiang ${ }^{2}$, S. Ciprini ${ }^{10}$, R. Claus ${ }^{2}$, \\ J. Cohen-Tanugi ${ }^{20}$, J. Conrad ${ }^{21,22,23}$, C. D. Dermer ${ }^{18}$, F. de Palma ${ }^{13,14}$, S. W. Digel ${ }^{2}$, P. S. Drell ${ }^{2}$, R. Dubois ${ }^{2}$, \\ C. Favuzzi ${ }^{13,14}$, E. C. Ferrara ${ }^{24}$, W. B. Focke 2 , Y. Fukazawa ${ }^{25}$, S. Funk ${ }^{2}$, P. Fusco ${ }^{13,14}$, F. Gargano ${ }^{14}$, \\ S. Germani ${ }^{9,10}$, N. Giglietto ${ }^{13,14}$, F. Giordano ${ }^{13,14}$, M. Giroletti ${ }^{26}$, T. Glanzman ${ }^{2}$, G. Godfrey ${ }^{2}$, I. A. Grenier ${ }^{4,1}$, \\ S. Guiriec ${ }^{27}$, D. Hadasch ${ }^{16}$, Y. Hanabata ${ }^{25}$, A. K. Harding ${ }^{24}$, K. Hayashi ${ }^{25}$, M. Hayashida ${ }^{2}$, R. E. Hughes ${ }^{12}$, \\ R. Itoh ${ }^{25}$, G. Jóhannesson ${ }^{2}$, A. S. Johnson ${ }^{2}$, W. N. Johnson ${ }^{18}$, T. Kamae ${ }^{2}$, H. Katagiri ${ }^{25}$, J. Kataoka ${ }^{28}$, \\ J. Knödlseder ${ }^{11}$, M. Kuss ${ }^{3}$, J. Lande ${ }^{2}$, L. Latronico ${ }^{3}$, S.-H. Lee ${ }^{2}$, M. Llena Garde ${ }^{21,22}$, F. Longo ${ }^{5,6}$, F. Loparco ${ }^{13,14}$, \\ M. N. Lovellette ${ }^{18}$, P. Lubrano ${ }^{9,10}$, A. Makeev ${ }^{18,19}$, P. Martin ${ }^{29}$, M. N. Mazziotta ${ }^{14}$, J. E. McEnery ${ }^{24,30}$, \\ J. Mehault ${ }^{20}$, P. F. Michelson ${ }^{2}$, T. Mizuno ${ }^{25,1}$, C. Monte ${ }^{13,14}$, M. E. Monzani ${ }^{2}$, A. Morselli ${ }^{31}$, I. V. Moskalenko ${ }^{2}$, \\ S. Murgia ${ }^{2}$, M. Naumann-Godo ${ }^{4}$, S. Nishino ${ }^{25}$, P. L. Nolan ${ }^{2}$, J. P. Norris ${ }^{32}$, E. Nuss ${ }^{20}$, T. Ohsugi ${ }^{33}$, A. Okumura ${ }^{34}$, \\ N. Omodei ${ }^{2}$, E. Orlando ${ }^{29}$, J. F. Ormes ${ }^{32}$, M. Ozaki ${ }^{34}$, D. Parent ${ }^{18,19}$, V. Pelassa ${ }^{20}$, M. Pepe ${ }^{9,10}$, M. Pesce-Rollins ${ }^{3}$, \\ F. Piron ${ }^{20}$, T. A. Porter ${ }^{2}$, S. Rainò ${ }^{13,14}$, R. Rando ${ }^{7,8}$, M. Razzano ${ }^{3}$, A. Reimer ${ }^{35,2}$, O. Reimer ${ }^{35,2}$, J. Ripken ${ }^{21,22}$, \\ T. Sada $^{25}$, H. F.-W. Sadrozinski ${ }^{36}$, C. Sgrò ${ }^{3}$, E. J. Siskind ${ }^{37}$, G. Spandre ${ }^{3}$, P. Spinelli ${ }^{13,14}$, M. S. Strickman ${ }^{18}$, \\ A. W. Strong ${ }^{29}$, D. J. Suson ${ }^{38}$, H. Takahashi ${ }^{33}$, T. Takahashi ${ }^{34}$, T. Tanaka ${ }^{2}$, J. B. Thayer ${ }^{2}$, D. J. Thompson ${ }^{24}$, \\ L. Tibaldo ${ }^{7,8,4,39,1}$, D. F. Torres ${ }^{16,40}$, A. Tramacere ${ }^{2,41,42}$, Y. Uchiyama ${ }^{2}$, T. Uehara ${ }^{25}$, T. L. Usher ${ }^{2}$, \\ J. Vandenbroucke ${ }^{2}$, V. Vasileiou ${ }^{43,44}$, N. Vilchez ${ }^{11}$, V. Vitale ${ }^{31,45}$, A. E. Vladimirov ${ }^{2}$, A. P. Waite ${ }^{2}$, P. Wang $^{2}$,

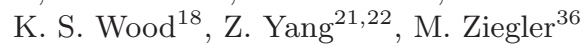

\begin{abstract}
We report an analysis of the interstellar $\gamma$-ray emission in the third Galactic quadrant measured by the Fermi Large Area Telescope. The window encompassing the Galactic plane from longitude $210^{\circ}$ to $250^{\circ}$ has kinematically well-defined segments of the Local and the Perseus arms, suitable to study the cosmic-ray densities across the outer Galaxy. We measure no large gradient with Galactocentric distance of the $\gamma$-ray emissivities per interstellar $\mathrm{H}$ atom over the regions sampled in this study. The gradient depends, however, on the optical depth correction applied to derive the H r column densities. No significant variations are found in the interstellar spectra in the outer Galaxy, indicating similar shapes of the cosmic-ray spectrum up to the Perseus arm for particles with $\mathrm{GeV}$ to tens of $\mathrm{GeV}$ energies. The emissivity as a function of Galactocentric radius does not show a large enhancement in the spiral arms with respect to the interarm region. The measured emissivity gradient is flatter than expectations based on a cosmic-ray propagation model using the radial distribution of supernova remnants and uniform diffusion properties. In this context, observations require a larger halo size and/or a flatter CR source distribution than usually assumed. The molecular mass calibrating ratio, $X_{\mathrm{CO}}=N\left(\mathrm{H}_{2}\right) / W_{\mathrm{CO}}$, is found to be $(2.08 \pm 0.11) \times 10^{20} \mathrm{~cm}^{-2}\left(\mathrm{~K} \mathrm{~km} \mathrm{~s}^{-1}\right)^{-1}$ in the Local-arm clouds and is not significantly sensitive to the choice of $\mathrm{H}$ I spin temperature. No significant variations are found for clouds in the interarm region.
\end{abstract}

Subject headings: cosmic rays - gamma rays: ISM - ISM: general 


$\begin{array}{lll}{ }^{1} \text { Corresponding authors: } & \text { I. } & \text { A. } \\ \text { nier, isabelle.grenier@cea.fr; } & \text { T. } & \text { Mizuno, }\end{array}$
mizuno@hep01.hepl.hiroshima-u.ac.jp; L. Tibaldo, luigi.tibaldo@pd.infn.it.

${ }^{2}$ W. W. Hansen Experimental Physics Laboratory, Kavli Institute for Particle Astrophysics and Cosmology, Department of Physics and SLAC National Accelerator Laboratory, Stanford University, Stanford, CA 94305, USA

${ }^{3}$ Istituto Nazionale di Fisica Nucleare, Sezione di Pisa, I-56127 Pisa, Italy

${ }^{4}$ Laboratoire AIM, CEA-IRFU/CNRS/Université Paris Diderot, Service d'Astrophysique, CEA Saclay, 91191 Gif sur Yvette, France

${ }^{5}$ Istituto Nazionale di Fisica Nucleare, Sezione di Trieste, I-34127 Trieste, Italy

${ }^{6}$ Dipartimento di Fisica, Università di Trieste, I-34127 Trieste, Italy

${ }^{7}$ Istituto Nazionale di Fisica Nucleare, Sezione di Padova, I-35131 Padova, Italy

${ }^{8}$ Dipartimento di Fisica "G. Galilei", Università di Padova, I-35131 Padova, Italy

${ }^{9}$ Istituto Nazionale di Fisica Nucleare, Sezione di Perugia, I-06123 Perugia, Italy

${ }^{10}$ Dipartimento di Fisica, Università degli Studi di Perugia, I-06123 Perugia, Italy

${ }^{11}$ Centre d'Étude Spatiale des Rayonnements, CNRS/UPS, BP 44346, F-30128 Toulouse Cedex 4, France

${ }^{12}$ Department of Physics, Center for Cosmology and Astro-Particle Physics, The Ohio State University, Columbus, OH 43210, USA

${ }^{13}$ Dipartimento di Fisica "M. Merlin" dell'Università e del Politecnico di Bari, I-70126 Bari, Italy

${ }^{14}$ Istituto Nazionale di Fisica Nucleare, Sezione di Bari, 70126 Bari, Italy

${ }^{15}$ Laboratoire Leprince-Ringuet, École polytechnique, CNRS/IN2P3, Palaiseau, France

${ }^{16}$ Institut de Ciencies de l'Espai (IEEC-CSIC), Campus UAB, 08193 Barcelona, Spain

${ }^{17}$ INAF-Istituto di Astrofisica Spaziale e Fisica Cosmica, I-20133 Milano, Italy

${ }^{18}$ Space Science Division, Naval Research Laboratory, Washington, DC 20375, USA

${ }^{19}$ George Mason University, Fairfax, VA 22030, USA

${ }^{20}$ Laboratoire de Physique Théorique et Astroparticules, Université Montpellier 2, CNRS/IN2P3, Montpellier, France

${ }^{21}$ Department of Physics, Stockholm University, AlbaNova, SE-106 91 Stockholm, Sweden

${ }^{22}$ The Oskar Klein Centre for Cosmoparticle Physics, AlbaNova, SE-106 91 Stockholm, Sweden

${ }^{23}$ Royal Swedish Academy of Sciences Research Fellow, funded by a grant from the K. A. Wallenberg Foundation

${ }^{24}$ NASA Goddard Space Flight Center, Greenbelt, MD 20771, USA

${ }^{25}$ Department of Physical Sciences, Hiroshima Univer-

\section{Introduction}

Knowledge of the distribution of cosmic-ray (CR) densities within our Galaxy is a key to understanding their origin and propagation. Highenergy CRs interact with the gas in the interstellar medium (ISM) or the interstellar radiation field, and produce $\gamma$-rays via nucleon-nucleon interac-

sity, Higashi-Hiroshima, Hiroshima 739-8526, Japan

${ }^{26}$ INAF Istituto di Radioastronomia, 40129 Bologna, Italy

${ }^{27}$ Center for Space Plasma and Aeronomic Research (CSPAR), University of Alabama in Huntsville, Huntsville, AL 35899, USA

${ }^{28}$ Research Institute for Science and Engineering, Waseda University, 3-4-1, Okubo, Shinjuku, Tokyo, 1698555 Japan

${ }^{29}$ Max-Planck Institut für extraterrestrische Physik, 85748 Garching, Germany

${ }^{30}$ Department of Physics and Department of Astronomy, University of Maryland, College Park, MD 20742, USA

${ }^{31}$ Istituto Nazionale di Fisica Nucleare, Sezione di Roma "Tor Vergata", I-00133 Roma, Italy

${ }^{32}$ Department of Physics and Astronomy, University of Denver, Denver, CO 80208, USA

${ }^{33}$ Hiroshima Astrophysical Science Center, Hiroshima University, Higashi-Hiroshima, Hiroshima 739-8526, Japan

${ }^{34}$ Institute of Space and Astronautical Science, JAXA, 3-1-1 Yoshinodai, Sagamihara, Kanagawa 229-8510, Japan

${ }^{35}$ Institut für Astro- und Teilchenphysik and Institut für Theoretische Physik, Leopold-Franzens-Universität Innsbruck, A-6020 Innsbruck, Austria

${ }^{36}$ Santa Cruz Institute for Particle Physics, Department of Physics and Department of Astronomy and Astrophysics, University of California at Santa Cruz, Santa Cruz, CA 95064, USA

${ }^{37}$ NYCB Real-Time Computing Inc., Lattingtown, NY 11560-1025, USA

${ }^{38}$ Department of Chemistry and Physics, Purdue University Calumet, Hammond, IN 46323-2094, USA

${ }^{39}$ Partially supported by the International Doctorate on Astroparticle Physics (IDAPP) program

${ }^{40}$ Institució Catalana de Recerca i Estudis Avançats (ICREA), Barcelona, Spain

${ }^{41}$ Consorzio Interuniversitario per la Fisica Spaziale (CIFS), I-10133 Torino, Italy

${ }^{42}$ INTEGRAL Science Data Centre, CH-1290 Versoix, Switzerland

${ }^{43}$ Center for Research and Exploration in Space Science and Technology (CRESST) and NASA Goddard Space Flight Center, Greenbelt, MD 20771, USA

${ }^{44}$ Department of Physics and Center for Space Sciences and Technology, University of Maryland Baltimore County, Baltimore, MD 21250, USA

${ }^{45}$ Dipartimento di Fisica, Università di Roma "Tor Vergata", I-00133 Roma, Italy 
tions, electron Bremsstrahlung and inverse Compton (IC) scattering. Since the ISM is transparent to these $\gamma$-rays, we can probe CRs in the local ISM, beyond direct measurements performed in the solar system, as well as in remote locations of the Galaxy. Although much effort has been made since the COS-B era (e.g., Strong et al. 1988; Strong \& Mattox 1996), the results have been limited by the angular resolution, effective area and energy coverage of the instruments. The advent of the Fermi Gamma-ray Space Telescope enables studying the spectral and spatial distribution of diffuse $\gamma$-rays and CRs with unprecedented sensitivity.

Here we report an analysis of diffuse $\gamma$-ray emission observed in the third Galactic quadrant. The window with Galactic longitude $210^{\circ} \leq l \leq 250^{\circ}$ and latitude $-15^{\circ} \leq b \leq+20^{\circ}$ hosts kinematically well-defined segments of the Local and the Perseus spiral arms and is one of the best regions to study the CR density distribution across the outer Galaxy. The region has been already studied by Digel et al. (2001) using EGRET data. The improved sensitivity and angular resolution of the Fermi LAT (Large Area Telescope; Atwood et al. 2009) and recent developments in the study of the ISM allow us to examine the CR spectra and density distribution with better accuracy. We exclude from the analysis the region of the Monoceros R2 giant molecular cloud and the Southern Filament of the Orion-Monoceros complex (e.g., Wilson et al. 2005), in $l \leq 222^{\circ}$ and $b \leq-6^{\circ}$, because 1) star forming activity and possible high magnetic fields suggested by the filamentary structure (e.g., Morris, Montani, \& Thaddeus 1980; Maddalena et al. 1986) could indicate a special $\mathrm{CR}$ environment, and 2) an $\mathrm{OB}$ association in Monoceros R2 may hamper the determination of ISM densities from dust tracers (see $\S 2.1 .2$ for details).

Study of the $X_{\mathrm{CO}}$ conversion factor which transforms the integrated intensity of the $2.6 \mathrm{~mm}$ line of carbon monoxide, $W_{\mathrm{CO}}$, into the molecular hydrogen column density, $N\left(\mathrm{H}_{2}\right)$, is also possible since the region contains well-known molecular complexes. In the Local arm we find the molecular clouds associated with Canis Major OB 1, NGC 2348 and NGC 2632 (Mel'nik \& Efremov 1995; Kaltcheva \& Hilditch 2000). At a few $\mathrm{kpc}$ from the Solar System, in the interarm, lower-density region located between the Local and Perseus arms, we find Maddalena's cloud (Maddalena \& Thaddeus 1985), a giant molecular cloud remarkable for its lack of star formation, and the cloud associated with Canis Major OB 2 (Kaltcheva \& Hilditch 2000).

This study complements the Fermi LAT study of the Cassiopeia and Cepheus region in the second quadrant reported by Abdo et al. (2010a). The paper is organized as follows. We describe the model preparation in $\S 2$ and the $\gamma$-ray observations, data selection and the analysis procedure in $\S 3$. The results are presented in $\S 4$, where we also discuss the emissivity profile measured for the atomic gas and we compare it with predictions by a CR propagation model. A summary of the study is given in $\S 5$.

\section{Modeling the Gamma-Ray Emission}

\subsection{Interstellar Gas}

\subsection{1. $\mathrm{H} \mathrm{I}$ and $\mathrm{CO}$}

In order to derive the $\gamma$-ray emissivities associated with the different components of the ISM we need to determine the interstellar gas column densities separately for each region and gas phase. For atomic hydrogen we used the Leiden/Argentine/Bonn Galactic H I survey by Kalberla et al. (2005). In order to turn the $\mathrm{HI}$ line intensities into $N\left(\mathrm{H}_{\mathrm{I}}\right)$ column densities, a uniform spin temperature $T_{\mathrm{S}}=125 \mathrm{~K}$ has often been adopted in previous studies. We will consider this option to directly compare our results with the former EGRET analysis of the same region (Digel et al. 2001) and other studies of the Galactic diffuse emission by the LAT (Abdo et al. 2009a, 2010a). Recent H I absorption studies (Dickey et al. 2009), however, point to larger average spin temperatures in the outer Galaxy, so we have tried different choices of $T_{\mathrm{S}}$ to evaluate how the optical depth correction affects the results. We will find that the emissivity per $\mathrm{H}$ I atom and the inferred CR density is affected by up to $\sim 50 \%$ in the Perseus arm, and will take this uncertainty into account in the discussion.

The integrated intensities of the $2.6 \mathrm{~mm}$ line of $\mathrm{CO}, W_{\mathrm{CO}}$, have been derived from the composite survey by Dame. Hartmann. \& Thaddeus (2001). The data have been filtered with the moment- 
masking technique in order to reduce the noise while keeping the resolution of the original data.

Figure 1 shows the velocity-longitude profile of $\mathrm{H}$ I emission in our region of interest (ROI). The preparation of maps accounting for the different Galactic structures present along the line of sight is similar to that described in detail in Abdo et al. (2010a) and based on a sequence of three steps:

1. preliminary separation within Galactocentric rings;

2. adjustment of the boundaries based on the velocity structures of the interstellar complexes;

3. correction for the spill-over due to the velocity dispersion of the broad $\mathrm{H}$ I lines between adjacent regions.

Four regions were defined in Galactocentric distance, namely the Local arm (Galactocentric radius $R \leq 10 \mathrm{kpc})$, the interarm region $(R=$ $10-12.5 \mathrm{kpc})$, the Perseus arm $(R=12.5-16 \mathrm{kpc})$ and the region beyond the Perseus arm (which hosts a faint segment of the outer arm; $R \geq$ $16 \mathrm{kpc})$. The boundaries separating these regions under the assumption of a flat rotation curve (Clemens 1985) for the case of $R_{0}=8.5 \mathrm{kpc}$ and $\theta_{0}=220 \mathrm{~km} \mathrm{~s}^{-1}$ (where $R_{0}$ and $\theta_{0}$ are the Galactocentric radius and the orbital velocity of the local group of stars, respectively) are overlaid in Figure 1.

The preparation of the $\mathrm{H}$ I and $\mathrm{CO}$ gas maps started from these preliminary velocity boundaries, which were then adjusted for each line of sight to the closest minimum in the $\mathrm{H}$ I spectrum 1. Then, the spill-over from one velocity interval to the next ones due to the velocity dispersion for the broad $\mathrm{H}_{\mathrm{I}}$ lines was corrected by fitting each $\mathrm{H}_{\mathrm{I}}$ spectrum with a combination of Gaussian profiles. We believe that this separation procedure provides more accurate estimates of the ISM column densities of each Galactic region than a simple slicing based on the rotation curve.

In particular, effort was put into separating the outer arm structures from the more massive Perseus arm component, especially at $l \gtrsim 235^{\circ}$

1 The minima are unlikely to be due to self absorption, because the velocity-distance relation is single valued in the outer Galaxy. where the $\mathrm{H}$ I lines from the two regions merge into a single broad component. For directions where a minimum in the $\mathrm{H}$ I brightness temperature profile was not found near the $R=16 \mathrm{kpc}$ velocity boundary, we integrated the profiles on both sides of the $R=16 \mathrm{kpc}$ velocity boundary to estimate the Perseus and outer arm contributions. Then we inserted a line in the Gaussian fitting at the outerarm velocity extrapolated from the $l-v$ trend observed at $l \gtrsim 235^{\circ}$ to correct for the spill-over due to the velocity dispersion. Given these difficulties we expect large systematic uncertainties in the outer-arm $N(\mathrm{H} \mathrm{I})$ column densities and the corresponding $\gamma$-ray emissivities will not be considered for the scientific interpretation. We note that the impact on the emissivities associated with the inner regions is small, $\leq 10 \%$ as described in $\S 4.3$.

The resulting maps are shown in Figure 2 and 3 They exhibit a low level of spatial degeneracy, and thus allow us to separate the $\gamma$-radiation arising from the interaction with CRs in each component.

\subsubsection{Interstellar reddening}

It has been long debated whether the combination of $\mathrm{H}$ I and $\mathrm{CO}$ surveys traces total column densities of neutral interstellar matter. By comparing gas line surveys, the $\gamma$-ray observations by EGRET and dust thermal emission, Grenier et al. (2005) reported a considerable amount of neutral gas at the interface between the two $\mathrm{H}$ I and $\mathrm{CO}$ emitting phases, associated with cold dust but not properly traced by $\mathrm{H}$ I and $\mathrm{CO}$ observations. Their finding was then confirmed by LAT data for the Gould Belt in the second quadrant Abdo et al. 2010a).

In order to complement the $\mathrm{HI}$ and $\mathrm{CO}$ maps, we have prepared a map derived from the $E(B-V)$ reddening map by Schlegel et al. (1998). The residual point sources at low latitudes were masked by setting to zero regions of $0.2^{\circ}$ radius centered on the positions of potential IRAS point sources 2 if the $E(B-V)$ magnitude exceeded by $\gtrsim 20 \%$ that in surrounding pixels. The masked regions were then restored through an inpainting technique (Elad et al. 2005). In the course of the work, various source masking techniques have 2 http://cdsarc.u-strasbg.fr/viz-bin/Cat?II/274 See
Beichman et al. (1988). 
been used with negligible impact on the $\mathrm{H}$ I and $\mathrm{CO}$ emissivity results.

The resulting map was fitted with a linear combination of the set of $N(\mathrm{HI})$ and $W_{\mathrm{CO}}$ maps described in $\S 2.1 .1$. The operation was repeated for different choices of $\mathrm{H}$ I spin temperature. The fit was performed over the same region as for the $\gamma$ ray analysis, excluding a $3^{\circ} \times 3^{\circ}$ region centered around Canis Major OB 1 (Mel'nik \& Efremov 1995) where the temperature correction applied by Schlegel et al. (1998) to construct the $E(B-V)$ map from the dust thermal emission is highly uncertain. A preliminary fit had led to extremely negative residuals $(\leq-1 \mathrm{mag})$ around $l=224^{\circ}$, $b=-3^{\circ}$. Therefore, the residual $E(B-V)$ map was calculated masking this region in the fit. We are aware that the temperature corrections used by Schlegel et al. (1998) are less reliable with decreasing latitude, but the improvement we find in the $\gamma$-ray fit by adding the dust residual map supports the use of their map at low latitude.

The residual $E(B-V)_{\text {res }}$ map, after subtracting the linear combination of $N(\mathrm{HI})$ and $W_{\mathrm{CO}}$ maps, is shown in Figure 4 (left panel). The residuals typically range from -0.5 to +0.5 magnitude. Large regions of positive residuals are found along the Galactic plane, in association with molecular/atomic clouds. They are expected to trace gas not correctly traced by $\mathrm{H}$ I and $\mathrm{CO}$ surveys. A remarkable region of positive residuals is detected at intermediate latitudes around $l=245^{\circ}$, $b=+17^{\circ}$, in a region not covered by $\mathrm{CO}$ surveys. It corresponds to positive residuals also in $\gamma$-rays ( $\S 3.2$ ) and may be due to a missing, but possibly CO-bright molecular cloud (already suggested by Dame, Hartmann, \& Thaddeus 2001 discussing the completeness of their survey, see Figure 8 of their paper). The negative residuals are generally small and may result from limitations in the gas column density derivation and/or dust spectral variations. The dust residual map compares well with the $\gamma$-ray residual map obtained when using only $\mathrm{H}$ I and $\mathrm{CO}$ to model the $\gamma$-ray emission (Figure 4, right panel). The correlation between the spatial distributions of the dust and $\gamma$-ray residuals is statistically confirmed in $\S 3.2$. Dust and $\gamma$-rays are consistent with the presence of missing gas in the positive residual clumps. The faint "glow" of negative residuals on both sides of the Galactic plane is driven by the nearby $N\left(\mathrm{H}_{\mathrm{I}}\right)$ maps and it remains even when using the smallest possible column-densities derived in the optically thin case. It may suggest a small change in average spin temperature from the massive, compact clouds sampled in the plane to the more diffuse envelopes sampled off the plane, or it may be due to the presence of more missing gas in the plane than our templates can provide for in the fit. The dust-to-gas ratio as well as the $\gamma$-ray emissivity in the $\mathrm{H}$ I components would then be driven to higher values by the low latitude data and would slightly overpredict the data off the plane.

The interpretation of the $E(B-V)_{\text {res }}$ map in this region of the sky is complicated by the lack of distance information for the dust emission. It is not possible to unambiguously assign the residuals to any of the regions under study. Since we aim at separating different regions along the lines of sight to investigate the CR density gradient in the outer Galaxy, using the $\mathrm{H}$ I and $\mathrm{CO}$ lines is essential. We have therefore used the $E(B-V)_{\text {res }}$ map to correct for the total gas column densities. This approach is supported by the correlation we find between the dust and $\gamma$-ray data $(\S 3.2)$. We also note that, since the dust contribution linearly correlated with the $\mathrm{HI}$ and $\mathrm{CO}$ maps has been removed in the $E(B-V)_{\text {res }}$ map, this procedure allows us to extract the $\gamma$-ray emissivities that are actually correlated with the $\mathrm{HI}$ and $\mathrm{CO}$ components.

\subsection{IC and Point Sources}

To model $\gamma$-ray emission not related with interstellar gas, we referred to the GALPROP code (e.g. Strong \& Moskalenko 1998; Strong et al. 2007) for $\gamma$-rays produced through IC scattering and to the first Fermi LAT catalog (1FGL) for point sources (Abdo et al. 2010b).

GALPROP 3 Strong \& Moskalenko 1998; Strong et al. 2007) is a numerical code which solves the CR transport equation within the Galaxy and predicts the $\gamma$-ray emission produced via interactions of CRs with interstellar matter (nucleon-nucleon interaction and electron Bremsstrahlung) and lowenergy photons (IC scattering). IC emission is calculated from the distribution of (propagated) electrons and the interstellar radiation fields de-

\footnotetext{
3 http://galprop.stanford.edu
} 
veloped by Porter et al. (2008). Here we adopt the IC model map produced in the GALPROP run 54_77Xvarh7S in which the CR electron spectrum is adjusted to agree with that measured by the LAT (Abdo et al. 2009b). This GALPROP model has been used in publications by the LAT collaboration such as Abdo et al. (2010c).

The 1FGL Catalog is based on the first 11 months of the science phase of the mission and contains 1451 sources detected at a significance $\gtrsim 4 \sigma$ (the threshold is 25 in term of test statistic, TS 4 ). For our analysis we considered 21 point sources in the ROI with TS larger than 50.

\subsection{Gamma-Ray Analysis Model}

Following a well-established approach that dates back to the COS-B era (e.g., Lebrun et al. 1983), we modeled the $\gamma$-ray emission as a linear combination of maps tracing the column density of the interstellar medium. This approach is based on a simple, but very plausible assumption: $\gamma$-rays are generated through interactions of CRs and the interstellar gas, and the ISM itself is transparent to $\gamma$-rays. Then, assuming that $\mathrm{CR}$ densities do not significantly vary over the scale of the interstellar complexes under study and that CRs penetrate clouds uniformly to their cores we can model the $\gamma$-ray intensities to first order as a linear combination of contributions from CR interactions with the different gas phases in the various regions along each line of sight.

We also added the IC model map by GALPROP and models for point sources taken from the 1FGL Catalog as described in $\S 2.2$. To represent the extragalactic diffuse emission and the residual instrumental background from misclassified CR interactions in the LAT detector, we also added an isotropic component. CR interactions with ionized gas are not explicitly included in the

4 The test statistic is defined as

$$
\mathrm{TS}=2\left(\ln L-\ln L_{0}\right)
$$

where $L$ and $L_{0}$ is the maximum likelihood with and without including the source in the model, respectively. $L$ is conventionally calculated as $\ln (L)=\Sigma_{i} n_{i} \ln \left(\theta_{i}\right)-\Sigma_{i} \theta_{i}$, where $n_{i}$ and $\theta_{i}$ are the data and the model-predicted counts in each pixel denoted by the subscript $i$, respectively (See, e.g., Mattox et al. 1996). TS is expected to be distributed as a $\chi^{2}$ with $n-n_{0}$ degrees of freedom if the numbers of free parameters in the model are respectively $n$ and $n_{0}$ ( 4 for sources in the 1FGL Catalog). model. The mass column densities of ionized gas are poorly known, but their contribution is generally lower $(\leq 10 \%)$ than that of the neutral gas and its scale height is much larger ( $\sim 1 \mathrm{kpc}$ compared with $\sim 0.2 \mathrm{kpc}$; Cordes \& Lazio 2002). We therefore expect the diffuse $\gamma$-ray emission originating from ionized gas to be largely accommodated in the fit by other components with large angular scales, such as the isotropic and IC ones, and to minimally impact the determination of the neutral gas emissivities.

Therefore, the $\gamma$-ray intensities $I_{\gamma}(l, b)\left(\mathrm{s}^{-1} \mathrm{~cm}^{-2} \mathrm{sr}^{-1} \mathrm{MeV}^{-1}\right)$ can be modeled as:

$$
\begin{aligned}
& I_{\gamma}(l, b)= \\
& \begin{aligned}
& \sum_{i=1}^{4} q_{\mathrm{HI}, i} \cdot N(\mathrm{HI})(l, b)_{i}+\sum_{i=1}^{3} q_{\mathrm{CO}, i} \cdot W_{\mathrm{CO}}(l, b)_{i} \\
&+q_{\mathrm{EBV}} \cdot \mathrm{E}(\mathrm{B}-\mathrm{V})_{\mathrm{res}}(l, b)+I_{\mathrm{IC}}(l, b) \\
&+I_{\text {iso }}+\sum_{j} \mathrm{PS}_{j}
\end{aligned}
\end{aligned}
$$

where sum over $i$ represents the combination of the Galactic regions, $q_{\mathrm{HI}, i}\left(\mathrm{~s}^{-1} \mathrm{sr}^{-1} \mathrm{MeV}^{-1}\right)$ and $q_{\mathrm{CO}, i}\left(\mathrm{~s}^{-1} \mathrm{~cm}^{-2} \mathrm{sr}^{-1} \mathrm{MeV}^{-1}\left(\mathrm{~K} \mathrm{~km} \mathrm{~s}^{-1}\right)^{-1}\right)$ are the emissivities per $\mathrm{HI}$ atom and per $W_{\mathrm{CO}}$ unit, respectively. $q_{\mathrm{EBV}}\left(\mathrm{s}^{-1} \mathrm{~cm}^{-2} \mathrm{sr}^{-1} \mathrm{MeV}^{-1} \mathrm{mag}^{-1}\right)$ is the emissivity per unit of the $E(B-V)_{\text {res }}$ map (for which independent normalizations are allowed between the positive and negative residuals; see $\S(3.2)$. $I_{\mathrm{IC}}$ and $I_{\text {iso }}$ are the IC model and isotropic background intensities $\left(\mathrm{s}^{-1} \mathrm{~cm}^{-2} \mathrm{sr}^{-1} \mathrm{MeV}^{-1}\right)$, respectively, and $\mathrm{PS}_{j}$ represents the point source contributions. Compared to the EGRET study by Digel et al. (2001), we use two additional maps to better trace the ISM: the CO map in the Perseus arm and the $E(B-V)_{\text {res }}$ map.

\section{Data Analysis}

\subsection{Observations and Data Selection}

The LAT on board the Fermi Gamma-ray Space Telescope, launched on 2008 June 11, is a pair-tracking telescope, detecting photons from $\sim 20 \mathrm{MeV}$ to more than $300 \mathrm{GeV}$. Details on the LAT instrument and pre-launch expectations of the performance can be found in Atwood et al. (2009), and the on-orbit calibration is described in Abdo et al. (2009c).

Routine science operations with the LAT started on 2008 August 4. We have accumulated events from 2008 August 4 to 2010 February 4 to 
study diffuse $\gamma$-rays in our ROI. During this time interval the LAT was operated in sky survey mode nearly all of the time, obtaining complete sky coverage every two orbits and relatively uniform exposures over time. We used the standard LAT analysis software, the Science Tools and selected events satisfying the standard low-background event selection (the so-called Diffuse class; Atwood et al. 2009) 5 We also required the reconstructed zenith angles of the arrival direction of photons to be less than $105^{\circ}$ and the center of the LAT field of view to be within $52^{\circ}$ from the zenith, in order to reduce the contamination of photons from the Earth limb. In addition, we excluded the period of time during which the LAT detected bright GRBs, i.e., GRB080916C (Abdo et al. 2009d), GRB090510 (Abdo et al. 2009e), GRB090902B (Abdo et al. 2009f), and GRB090926A (Abdo et al. 2010e).

\subsection{Analysis Procedure}

The model described by Equation (1) was fitted to the data using the Science Tools, which take into account the energy-dependent instrument point spread function and effective area. We have analyzed the LAT data from $100 \mathrm{MeV}$ to $25.6 \mathrm{GeV}$ using 13 logarithmically-spaced energy bands from $100 \mathrm{MeV}$ to $9.05 \mathrm{GeV}$, and a single band above $9.05 \mathrm{GeV}$. We then have compared the model and data in each energy band using a binned maximum-likelihood method with Poisson statistics (in $0.25^{\circ} \times 0.25^{\circ}$ bins); we thus did not assume an a-priori spectral shape of each model component except for the IC emission. For the other components the convolution with the instrument response functions was performed assuming an $E^{-2}$ spectrum, and the integrated intensities were allowed to vary in each narrow energy bin. Changing the fixed spectral shape index over the range from -1.5 to -3.0 has a negligible effect on the obtained spectrum. In the highest energy band, we have set both the normalization and the spectral index free to accommodate the wider bin width. We used a post-launch response function, P6_V3_DIFFUSE, developed to account for the $\gamma$ ray detection inefficiencies due to pile-up and acci-

5 Data and software are publicly available from the Fermi Science Support Center (http://fermi.gsfc.nasa.gov/ssc/). For this analysis we used the P6 Diffuse selection and the Science Tools version v9r16p0. dental coincidence in the LAT (Rando et al. 2009). We stopped at $25.6 \mathrm{GeV}$ since the photon statistics does not allow us to reliably separate different gas components above this energy.

We started with point sources detected with high significance (TS $\geq 100$ ) in the $1 F G L$ Catalog; we have 14 sources in our ROI for which the normalizations are set free. We also included 8 sources lying just outside $\left(\leq 5^{\circ}\right)$ of the region boundaries, with all the spectral parameters fixed to those in the 1FGL Catalog. As a starting point we used H I maps prepared for $T_{\mathrm{S}}=125 \mathrm{~K}$. We added model components step by step as described below.

We first fitted the LAT data using Equation (1) without the $E(B-V)_{\text {res }}$ map and the CO map in the Perseus arm, and then included the CO map and confirmed that the fit improved significantly; the test statistic summed over 14 bands with separate fits in each band (i.e., 14 more free parameters) is 187.6. The $\gamma$-ray emission associated to the gas traced by $\mathrm{CO}$ in the Perseus arm is thus significantly detected by the LAT.

Next we included the $E(B-V)_{\text {res }}$ map in the analysis. We allowed the independent normalizations between the positive part and the negative part of the $E(B-V)_{\text {res }}$ map, and found that the normalizations differ with each other. We thus will use the independent normalizations hereafter. We chose this model to better represent the LAT data and constrain the $\mathrm{CR}$ distributions, and leave a detailed discussion about the use of dust as ISM tracer to a dedicated paper. The improvement of the fit is very significant: TS $=1119.6$ for 28 more free parameters. The correlation between the $E(B-V)_{\text {res }}$ map and the $\gamma$-ray residual map obtained by the fit without the $E(B-V)_{\text {res }}$ map, shown in Figure 4 further supports the use of $E(B-V)_{\text {res }}$ map in our analysis.

We also tried a fit without the IC component to assess the systematics. The effects on the derived emissivities are typically $2-3 \%$ and $\sim 5 \%$ for $q_{\mathrm{HI}}$ and $q_{\mathrm{CO}}$, respectively. They are much smaller than the statistical errors and systematic uncertainties (see below), although the inclusion of the IC map improves the fit to the LAT data. Therefore the uncertainties on the IC model have no significant impact on our analysis due to its rather flat distribution across the region of interest while the gas in the ISM is highly structured. On 
the other hand, lowering the threshold for point sources down to $\mathrm{TS}=50$ yields an about twice smaller emissivity for the $W_{\mathrm{CO}}$ map in the Perseus arm. The emissivities of other components are unchanged within the statistical errors. This is plausibly due to the very clumpy distribution of the clouds in the Perseus arm as seen by a terrestrial observer, see Figure 3. which makes it difficult to separate from that of some discrete sources. We thus use Equation (1) with point sources detected at $\mathrm{TS} \geq 50$ in the $1 \mathrm{FGL}$ Catalog 6 as our baseline model, but we do not consider the highly uncertain CO emissivities in the Perseus arm for the discussion.

We summarize the results in Tables 1 and 2 for $T_{\mathrm{S}}=125 \mathrm{~K}$ and $250 \mathrm{~K}$, respectively, and the number of counts in each energy bin in Table 3 . The differential emissivities are multiplied by $E^{2}$ where $E$ is the center of each energy bin in logarithmic scale. They are given for each model component. We note that our isotropic term $\left(I_{\text {iso }}\right)$ includes the contribution of the instrumental background and might partially account also for ionized gas (see $\S 2.3$, thus it is significantly larger than the extragalactic diffuse emission reported by Abdo et al. (2010d).

To illustrate the fit quality, we give the data and model count maps and the residual map in Figure 5 (for $T_{\mathrm{S}}=125 \mathrm{~K}$ ), in which residuals (data minus model) are expressed in approximate standard deviation units (square root of model-predicted counts). Although some structures (clustering of positive or negative residuals) are observed, the map shows no excesses below $-4 \sigma$ and above $6 \sigma$. Over $99 \%$ of the pixels are within $\pm 3 \sigma$. We thus conclude that our model reasonably reproduces the data.

Figure 6] presents the fitted spectra for each component. The emission from the H I gas dominates the $\gamma$-ray flux. Although the emission from the gas in the CO-bright phase and that traced by $E(B-V)_{\text {res }}$ is fainter than the IC and isotropic components, their characteristic spatial structures (see Figure 2 and 3) allow their spectra to be reliably constrained.

To examine the effect of the optical depth cor-

6 Spectral parameters of point sources of $\mathrm{TS}=50-100$ are fixed to those given in the 1 FGL Catalog in the highest energy bin. $(9.05-25.6 \mathrm{GeV})$ rection applied to derive the $\mathrm{H}$ I maps, as anticipated above we tried several choices of a uniform $T_{\mathrm{S}}$. We stress that the true $T_{\mathrm{S}}$ is likely to vary within clouds, but we stick to this simple approximation exploring the following values: $100 \mathrm{~K}$ (which is a reasonable lower limit in the uniform approximation) $7,250 \mathrm{~K}$ and $400 \mathrm{~K}$ (which are the two values indicated by absorption measurements in the outer Galaxy by Dickey et al. 2009), and the optically-thin approximation (which yields the lower limit allowed on the atomic column densities). The results on the maximum loglikelihood values are summarized in Table 4 together with the integrated $\mathrm{HI}$ emissivities obtained above $100 \mathrm{MeV}$ in each region. The evolution of $\ln (L)$ with $T_{\mathrm{S}}$ is plotted in Figure 7 The H I emissivity varies by $+15 /-10 \%$ for the Local arm, $+10 /-0 \%$ for the interarm region, and $+50 /-25 \%$ for the Perseus arm with respect to the $T_{\mathrm{S}}=125 \mathrm{~K}$ case. We observe an increase of $\ln (L)$ with increasing spin temperature. Considering the fact that $T_{\mathrm{S}}=250 \mathrm{~K}$ is a typical value in the second quadrant of the outer Galaxy according to a recent study by Dickey et al. (2009) and because $\ln (L)$ saturates at $T_{\mathrm{S}} \geq 250 \mathrm{~K}$, we regard $250 \mathrm{~K}$ as a plausible estimate of the average $T_{\mathrm{S}}$ in our ROI. Unfortunately, the estimates by Dickey et al. (2009) have a rather large uncertainty (about $\pm 50 \mathrm{~K}$ ) in each Galactocentric radius bin, and they do not cover the region in the third quadrant we are investigating (see Figure 5 of Dickey et al. 2009). In the following sections, we will concentrate on $T_{\mathrm{S}}=125 \mathrm{~K}$ for comparison with previous $\gamma$-ray measurements and on $T_{\mathrm{S}}=250 \mathrm{~K}$ which agrees well with $\mathrm{H}$ I absorption and the LAT data.

\section{Results and Discussions}

\subsection{Emissivity Spectra of Atomic Gas}

In Figures 8 and 9 (left panels), we report the emissivity spectra found per $\mathrm{H}$ atom in the Local arm, interarm, Perseus arm and outermost regions for $T_{\mathrm{S}}=125$ and $250 \mathrm{~K}$, respectively. For comparison with the local interstellar spectrum (LIS) we also plot the model spectrum used in Abdo et al.

\footnotetext{
7 A truncation at $95 \mathrm{~K}$ was applied for channels where the brightness temperature was larger.

8 Note that, however, the data used by Dickey et al. (2009) do not cover the third Galactic quadrant
} 
(2009a) which agrees well with LAT data in a midto-high-latitude region $\left(22^{\circ} \leq|b| \leq 60^{\circ}\right)$ of the third quadrant (assuming $T_{\mathrm{S}}=125 \mathrm{~K}$ ). We see that the spectral shape of the Local-arm emissivity agrees well with the model for the LIS and does not depend on the choice of spin temperature. The integral emissivity of the Local arm is $10 \%$ lower than that reported by Abdo et al. (2009a) for the same spin temperature. This difference is not significant given the uncertainties in the kinematic separation of the gas components. The present result is also consistent with the measurement in the second quadrant Abdo et al. 2010a). Together they show that the CR density along the Local arm is rather uniform within $1 \mathrm{kpc}$ around the Sun, both in the second and third quadrants.

The comparison of the data with the model emissivity expected for the Local arm region based on locally-measured CRs (Figures 8 and 9) indicates a better fit for higher $T_{\mathrm{S}} ; T_{\mathrm{S}}=125 \mathrm{~K}$ gives emissivities 15-20\% lower than the model, whereas $T_{\mathrm{S}}=250 \mathrm{~K}$ shows better agreement by about $10 \%$. Although the theoretical emissivity has uncertainties due to imperfect knowledge of the CR spectrum (see Abdo et al. 2009a), the fact that a high $T_{\mathrm{S}}$ value yields a better match both to the local absolute emissivity and to the spatial distribution of the diffuse emission (Figure [7) leads to larger $T_{\mathrm{S}}$ than a value conventionally used in $\gamma$-ray astrophysics $(125 \mathrm{~K})$. This is in accord with independent estimates of $T_{\mathrm{S}}$ as discussed in $\S 3.2$

We also observe that the emissivity spectra do not vary significantly with Galactocentric distances in the outer Galaxy. To examine the spectral shape more quantitatively, we present the emissivity ratios of the interarm and Perseus regions relative to the Local arm in the right panels of Figures 8 and 9. The spectral shape in the interarm region is found to be consistent with that in the Local arm; a fit to the data for $T_{\mathrm{S}}=125 \mathrm{~K}$ with a constant ratio gives $\chi^{2}=7.3$ for 13 degrees of freedom. Although the fit is not fully acceptable for the Perseus arm $\left(\chi^{2}=24.3\right)$, the large $\chi^{2}$ is driven solely by the last bin. We note a possible interplay between the Perseus-arm and the adjacent outer-arm emissivities in the highest energy bins (see left panels of Figure 8 and 91). It can be due to a small but non-negligible spatial difference between the modeled templates and data and/or to the presence of unresolved point sources (gener- ally harder than diffuse emission). Photon fluctuations from the structured gas components can also lead the fit to a slightly different solution in the spatial separation of the components. One would expect these possible systematic uncertainties to become important at high energy given the limited counts in the overall map. It is difficult to quantitatively test these effects without knowledge of the true model distributions, but we can note that the small deviations seen at 400-560 MeV and 1.6$2.2 \mathrm{GeV}$ from a constant ratio are not confirmed by the general trend of the other points. They indicate that there are systematic uncertainties not fully accounted for by the statistical errors in the fit. We thus do not claim nor deny the spectral softening of the Perseus arm at high energy. A test using $T_{\mathrm{S}}=250 \mathrm{~K}$ for the $N(\mathrm{HI})$ maps gives the same conclusion on the spectral shape. We thus conclude that the spectral shapes are consistent with the LIS in the $0.1-6 \mathrm{GeV}$ energy band, independent of the assumed $T_{\mathrm{S}}$. Considering that these $\gamma$-rays trace CR nuclei of energies from a few $\mathrm{GeV}$ to about $100 \mathrm{GeV}$ (see, e.g., Figure11 of Mori 1997), LAT data indicate that the energy distribution of the main component of Galactic CRs does not vary significantly in the outer Galaxy in the third quadrant. We note that Abdo et al. (2010a) reported a possible spectral hardening in the Perseus arm in the second quadrant. This might be due to the presence of the very active star-forming region of NGC 7538 and of CRs having not diffused far from their sources, or to contamination by hard unresolved point sources. In fact Abdo et al. (2010a) did not rule out the possibility that their result is due to systematic effects.

\subsection{Calibration of Molecular Masses}

High-energy $\gamma$-rays are a powerful probe to determine the $\mathrm{CO}$-to- $\mathrm{H}_{2}$ calibration ratio, $X_{\mathrm{CO}}$, if the CR flux is comparable in the different gas phases inside a cloud. Since the $\gamma$-ray emission from the molecular gas is primarily due to CR interactions with $\mathrm{H}_{2}$, and since the molecular binding energy is negligible in processes leading to $\gamma$ ray production, the emissivity per $\mathrm{H}_{2}$ molecule is expected to be twice the emissivity per $\mathrm{H}$ I atom. Then, under the hypothesis that the same CR flux penetrates the $\mathrm{H}$ I- and CO-bright phases of an interstellar complex, we can calculate $X_{\mathrm{CO}}$ as $q_{\mathrm{CO}}=2 X_{\mathrm{CO}} \cdot q_{\mathrm{HI}}$. 
We show $q_{\mathrm{CO}}$ as a function of $q_{\mathrm{HI}}$ for the Local arm and the interarm region in Figure 10. We do not consider the correlation in the Perseus arm, because $q_{\mathrm{CO}}$ from this region is affected by large systematic uncertainties (see $\S 3.2$ ). Since the emissivity associated with the CO-bright gas is not well determined in the lowest energy range (see Table 1 and 2) because of the poor angular resolution of the LAT, and the fit at very high energy is affected by larger uncertainties ( $\S 4.1)$, we have plotted only data in the $200 \mathrm{MeV}-9.05 \mathrm{GeV}$ range. The linear correlation supports the assumption that Galactic CRs penetrate molecular clouds uniformly to their cores. It also suggests that contamination from point sources and CR spectral variations within the clouds are small.

We have derived the maximum-likelihood estimates of the slope and intercept of the linear relation between $q_{\mathrm{CO}}$ and $q_{\mathrm{HI}}$ taking into account that $q_{\mathrm{CO}}$ and $q_{\mathrm{HI}}$ are both measured (not true) values with known uncertainties. The resulting intercepts are compatible with zero. The $X_{\mathrm{CO}}$ values we have obtained for $T_{\mathrm{S}}=250 \mathrm{~K}$ are $(2.08 \pm 0.11) \times$ $10^{20} \mathrm{~cm}^{-2}\left(\mathrm{~K} \mathrm{~km} \mathrm{~s}^{-1}\right)^{-1}$ for the Local arm $(R \leq$ $10 \mathrm{kpc})$ and $(1.93 \pm 0.16) \times 10^{20} \mathrm{~cm}^{-2}(\mathrm{~K} \mathrm{~km} / \mathrm{s})^{-1}$ for the interarm region $(R=10-12.5 \mathrm{kpc})$. Decreasing the spin temperature to $125 \mathrm{~K}$ does not affect the $X_{\mathrm{CO}}$ derivation in the well resolved, not too massive, clouds of the Local arm where we find $X_{\mathrm{CO}}=(2.03 \pm 0.11) \times 10^{20} \mathrm{~cm}^{-2}\left(\mathrm{~K} \mathrm{~km} \mathrm{~s}^{-1}\right)^{-1}$. On the other hand, the separation in the $\gamma$-ray fit between the dense $\mathrm{H}$ i peaks and clumpy $\mathrm{CO}$ cores becomes more difficult for more distant, less resolved clouds where $\mathrm{HI}$ and $\mathrm{CO}$ tend to peak in the same directions. A change in the largest $N(\mathrm{HI})$ column-densities from the optical depth correction can impact the $X_{\mathrm{CO}}$ determination in two ways: first by changing the $q_{\mathrm{HI}}$ emissivity, second by modifying the $N(\mathrm{HI})$ contrast within the cloud, hence the $\mathrm{H}$ I and CO separation. The global impact is mild since we find $X_{\mathrm{CO}}=(1.56 \pm 0.17) \times 10^{20} \mathrm{~cm}^{-2}\left(\mathrm{~K} \mathrm{~km} \mathrm{~s}^{-1}\right)^{-1}$ in the interarm region for $T_{\mathrm{S}}=125 \mathrm{~K}$.

Abdo et al. (2010a) reported comparable values of $X_{\mathrm{CO}}$ in the second quadrant for $T_{\mathrm{S}}=125 \mathrm{~K}$ : they obtained $(1.59 \pm 0.17) \times 10^{20} \mathrm{~cm}^{-2}\left(\mathrm{~K} \mathrm{~km} \mathrm{~s}^{-1}\right)^{-1}$ and $(1.9 \pm 0.2) \times 10^{20} \mathrm{~cm}^{-2}(\mathrm{~K} \mathrm{~km} / \mathrm{s})^{-1}$ for the Local arm $(R \leq 10 \mathrm{kpc})$ and the Perseus arm. Given the systematic uncertainty in $X_{\mathrm{CO}}$, roughly of the order of $0.3 \times 10^{20} \mathrm{~cm}^{-2}\left(\mathrm{~K} \mathrm{~km} \mathrm{~s}^{-1}\right)^{-1}$, due to $\mathrm{H}$ I optical depth correction, the results of both studies point to a rather uniform ratio over several kpc in the outer Galaxy. Yet, these values are twice larger than found in the very nearby Gould-Belt clouds of Cassiopeia and Cepheus, $X_{\mathrm{CO}}=(0.87 \pm 0.05) \times 10^{20} \mathrm{~cm}^{-2}(\mathrm{~K} \mathrm{~km} / \mathrm{s})^{-1}$, $T_{\mathrm{S}}=125 \mathrm{~K}$. However, we confirm that the increase in $X_{\mathrm{CO}}$ beyond the solar circle is significantly lower than the trend adopted in the model of Strong et al. (2004b). What fraction of the Gould-Belt to Local-arm difference in the average $X_{\mathrm{CO}}$ can be attributed to a difference in the spatial sampling (resolution) of the clouds remains to be investigated.

Nearly the same region has been analyzed by Digel et al. (2001) using EGRET data. The main difference from their analysis is our improved scheme for the kinematical separation of the ISM components along the lines of sight and the inclusion of the reddening residual map. The $X_{\mathrm{CO}}$ value measured in the Local arm by EGRET, $(1.64 \pm 0.31) \times 10^{20} \mathrm{~cm}^{-2}\left(\mathrm{~K} \mathrm{~km} \mathrm{~s}^{-1}\right)^{-1}$ (for $T_{\mathrm{S}}=125 \mathrm{~K}$ ), is statistically compatible with ours. The fact that we excluded the region of Monoceros R2 from the analysis can also explain in part this difference.

Because of the pile-up of different clouds along a line of sight, the derivation of individual cloud masses is beyond the scope of this study. Let us just note that Maddalena's cloud, with its very low rate of star formation, seems to share a quite conventional $X_{\mathrm{CO}}$ factor. Further investigation, including higher resolution $\gamma$-ray maps when more high-energy LAT data become available, is required to fully understand the mass distribution in the clouds.

\subsection{The Gradient of CR Densities beyond the Solar Circle}

In Figure11 (left panel) we show the emissivity gradient found beyond the Solar circle for different spin temperatures. Here we do not include the results for the optically-thin approximation which is equivalent to an infinitely high $T_{\mathrm{S}}$ and gives similar emissivities to $T_{\mathrm{S}}=400 \mathrm{~K}$. The typical statistical errors associated with these measurements are illustrated in the right panel for the $T_{\mathrm{S}}=125 \mathrm{~K}$ case. In the right panel, a shaded area shows the characteristic systematic error due to the LAT event selection efficiency, evaluated to be $\sim 10 \%$ 
in the energy range under study.

In order to evaluate the impact of the delicate separation of the gas in the outermost region, we have compared two extreme cases. The first one adopts the kinematic $R=16 \mathrm{kpc}$ boundary and applies no correction for velocity dispersion, the second assigns all the outer-arm gas to the Perseus arm. The emissivity in the Perseus arm differs by about $5 \%$ from the original one, and those in the Local arm and interarm regions hardly change. Therefore these effects are significantly smaller than the uncertainties due to the optical depth correction of the $\mathrm{H}$ I data. We also note that the main effect of the LAT selection efficiency uncertainty is to rigidly shift the profile without any significant impact on the gradient.

We thus conclude that the most important source of uncertainty in the $\mathrm{CR}$ density gradient derivation is currently that in the $N(\mathrm{H} \mathrm{I})$ determination. This is mainly because the optical depth correction is larger for dense $\mathrm{H}$ I clouds in the Local and Perseus arms than for diffuse clouds in the interarm region. The loss in contrast between the dense (low-latitude) and more diffuse (midlatitude) $\mathrm{H}$ I structures resulting from an increase in spin temperature affects the fit, particularly in the Perseus component which is more narrowly concentrated near the plane. When probing the CR densities as the "ratio" between the observed numbers of $\gamma$-rays to $\mathrm{H}$ atoms, at the precision provided by the LAT the uncertainties in the ISM densities are dominant.

\subsubsection{Comparison with EGRET and the arm/interarm contrast}

An interesting finding of the former EGRET analysis (Digel et al. 2001) was an enhancement of the $\gamma$-ray emissivity in the Perseus arm compared with the interarm region. This possibility is relevant for models of diffuse $\gamma$-ray emissions based on the assumption that CR and ISM densities are coupled (e.g., Hunter et al. 1997, and references therein).

The Local arm emissivity obtained by the EGRET study for $T_{\mathrm{S}}=125 \mathrm{~K}$ is (1.81 \pm $0.17) \times 10^{-26}$ photons s ${ }^{-1} \mathrm{sr}^{-1} \mathrm{H}$-atom ${ }^{-1}$, which is $\sim 25 \%$ larger than our LAT result. However, the two studies are based on different $\mathrm{H}$ I surveys which yield different total $N(\mathrm{H}$ I) column densities integrated along the lines of sight. The columndensity ratios between the surveys varies from 0.6 to 1.0 within the ROI, with an average value of $\sim 0.8$. The difference is likely due to the improved correction for stray-radiation in the more recent survey, as discussed in Kalberla et al. (2005). The EGRET Local-arm emissivity scaled by 0.8 is in good agreement with our result for the same spin temperature. If we do not include the $E(B-V)_{\text {res }}$ map in the fitting, we obtain an emissivity of $(1.68 \pm 0.05) \times 10^{-26}$ photons s ${ }^{-1} \mathrm{sr}^{-1} \mathrm{H}_{\text {-atom }}{ }^{-1}$ which is still consistent with the down-scaled EGRET result within $\sim 15 \%$. We thus conclude that our result is consistent with the previous study but is more reliable because of higher $\gamma$-ray statistics, finer resolution and an improved $\mathrm{H}$ I gas survey.

We can therefore compare the present emissivity gradient (for consistency in the case $T_{\mathrm{S}}=$ $125 \mathrm{~K}$ ) with that reported by the EGRET study, as summarized in Figure 11 (right panel in which the EGRET results multiplied by 0.8 are also shown). Although we observe good agreement between the two studies in the Local and the Perseus arms, this is not true for the arm/interarm contrast. The difference could be due to the simple partitioning in cloud velocity used for the EGRET study. The H I mass obtained for clouds in the interarm region with the simple partitioning is $20 \%-40 \%$ larger (for $T_{\mathrm{S}}=125 \mathrm{~K}$ ) than with our separation scheme, exaggerating the amount of gas in the interarm region, and thus lowering the emissivity by the same amount. Our emissivity profile is thus consistent with the previous study, but with improved precision (smaller statistical errors) and accuracy (more reliable region separation method and better estimation of the point source contributions). We thus do not confirm a marked drop in the interarm region.

Low spin temperatures yield a smooth decline in $\mathrm{H}$ I emissivity to $R \simeq 16 \mathrm{kpc}$ in the outer Galaxy, without showing a significant coupling with ISM column densities. The Perseusto-interarm contrast is at most of the order of 15\%-20\% for high spin temperatures as shown in the left panel of Figure 11. These profiles are similar at all energies, in particular at high energies where the component separation is more reliable thanks to the better angular resolution. The surface density of $\mathrm{H}$ I in the Perseus arm is 
on average $30 \%-40 \%$ higher than in the interarm region. Therefore, even if we adopt $T_{\mathrm{S}}=400 \mathrm{~K}$ which gives the largest arm-interarm contrast, the coupling scale (or the coupling length) between the CRs and matter (e.g., Hunter et al. 1997) required to agree with the LAT data would be larger than those usually assumed for this type of model ( $\sim 2$ kpc. See e.g., Digel et al. 2001, Figure 7). Whether the true emissivity profile exhibits a small contrast between the arms or smoothly declines with distance is beyond our measurement capability without further constraints on the $\mathrm{H}$ I column-density derivation. New H I absorption measurements will allow us to investigate this issue with better accuracy.

\subsubsection{Comparison with a Propagation Model: the CR gradient problem}

To compare with the second quadrant results (Abdo et al. 2010a), we have integrated the emissivities above $200 \mathrm{MeV}$ for $T_{\mathrm{S}}=125 \mathrm{~K}$. We find values of $(0.817 \pm 0.016) \times 10^{-26}$ photons s${ }^{-1} \mathrm{sr}^{-1} \mathrm{H}$-atom $(0.705 \pm 0.018) \times 10^{-26}$ photons s${ }^{-1} \mathrm{sr}^{-1} \mathrm{H}$-atom ${ }^{-1}$ and $(0.643 \pm 0.022) \times 10^{-26}$ photons s ${ }^{-1} \mathrm{sr}^{-1} \mathrm{H}_{\text {-atom }}{ }^{-1}$ for the Local-arm, interarm, and Perseus-arm regions, respectively. The nearer value is about $20 \%$ lower than in the 2nd quadrant (which, however, samples very nearby clouds in the Gould Belt) and the outer ones compare very well with the $2 \mathrm{nd}$ quadrant measurement over the same Galactocentric distance range. Despite the uncertainties due to the optical-depth correction (that might have a different impact in the two quadrants), both LAT studies consistently point to a slowly decreasing emissivity profile beyond $R=10 \mathrm{kpc}$.

Let us consider the predictions by a CR propagation model to see the impact of such a flat profile on the CR source distribution and propagation parameters. We adopted a GALPROP model, starting from the configuration used for the run 54_77Xvarh7S which we used to predict the IC contribution. The CR source distribution in this model is,

$$
f(R)=\left(\frac{R}{R_{\odot}}\right)^{1.25} \exp \left(-3.56 \cdot \frac{R-R_{\odot}}{R_{\odot}}\right),
$$

where $R_{\odot}=8.5 \mathrm{kpc}$ is the distance of the Sun to the Galactic center. As shown in Figure 12 this function is intermediate between the distribution of supernova remnants (SNRs) obtained from the $\Sigma-D$ relation (Case \& Bhattacharva 1998) and that traced by pulsars (Lorimer 2004). The boundaries of the propagation region are chosen to be $R_{\mathrm{h}}=30 \mathrm{kpc}$ (maximum Galactocentric radius) and $z_{\mathrm{h}}=4 \mathrm{kpc}$ (maximum height from the Galactic plane), beyond which free escape is assumed. The spatial diffusion coefficient is assumed to be uniform across the Galaxy and is taken as $D_{x x}=\beta D_{0}(\rho / 4 \mathrm{GV})^{\delta}$, where $\beta \equiv v / c$ is the velocity of the particle relative to the speed of light and $\rho$ is the rigidity. We adopted $D_{0}=5.8 \times 10^{28} \mathrm{~cm}^{2} \mathrm{~s}^{-1}$ and $\delta=0.33$ (Kolmogorov spectrum). Reacceleration due to the interstellar magnetohydrodynamic turbulence, which is thought to reproduce the observed B/C ratio at low energy, assumes an Alfvén velocity $v_{\mathrm{A}}=30 \mathrm{~km} \mathrm{~s}^{-1}$. The $\mathrm{CR}$ source distribution and propagation model parameters have been used often in the literature (see e.g., Strong et al. 2004a). We note that the same CR source distribution and similar propagation parameters are adopted in the GALPROP run used by Abdo et al. (2010a).

The left panel of Figure 13 compares the calculated profile (solid line) with LAT constraints (bow-tie plot bracketing the profiles obtained for different $T_{\mathrm{S}}$; see the left panel of Figure 11). The model is normalized to the LAT measurement in the innermost region. Despite the large uncertainties, LAT data lead to a significantly flatter profile than predicted by our model; the LAT results indicate to a factor of 2 larger emissivity (CR energy density) in the Perseus arm even if we assume $T_{\mathrm{S}}=100 \mathrm{~K}$. The higher $T_{\mathrm{S}}$ makes the discrepancy larger, hence the conclusion is robust. Not using the $E(B-V)_{\text {res map in the analysis does }}$ not change the conclusion, since the emissivities in the interarm region and the Perseus arm are almost unaffected by its presence.

The discrepancy between the $\gamma$-ray emissivity gradient in the Galaxy and the distribution of putative CR sources has been known as the "gradient problem" since the COS-B era (e.g., Bloemen 1989). It has led to a number of possible interpretations, including, for the specific case of the outer Galaxy, the possibility of a very steep gradient in $X_{\mathrm{CO}}$ beyond the solar circle (Strong et al. 2004b). The emissivities in the outer Galaxy were more difficult to determine in the COS-B/EGRET era due to lower statistics and higher backgrounds. Now, thanks to the high quality of the LAT data and the 
improved component separation technique applied to gas line data, we measure a flat $\mathrm{H}$ I emissivity gradient in the outer Galaxy together with a flat evolution of $X_{\mathrm{CO}}$ over several kpc, so the gradient problem requires another explanation.

The most straightforward possibility is a larger halo size $\left(z_{\mathrm{h}}\right)$, as discussed by, e.g., Stecker \& Jones (1977), Bloemen (1989) and Strong \& Moskalenko (1998). We therefore tried several choices of $z_{\mathrm{h}}$ and $D_{0}$ as summarized in the dotted lines in the same figure. The values of $D_{0}$ are chosen to reasonably reproduce the LIS of protons and electrons, $\mathrm{B} / \mathrm{C}$ ratio and ${ }^{10} \mathrm{Be} /{ }^{9} \mathrm{Be}$ ratio at the solar system, and are similar to those given in Strong \& Moskalenko (1998). All models are normalized to the LAT data in the Local arm. Models with $z_{\mathrm{h}}=4 \mathrm{kpc}$ or smaller are found to give too steep emissivity gradients. A CR source distribution as in equation 2 with a very large halo $\left(z_{\mathrm{h}} \geq 10 \mathrm{kpc}\right)$ provides a gradient compatible with the $\gamma$-ray data, if we fully take into account the systematic uncertainties. We note that $z_{\mathrm{h}}=10 \mathrm{kpc}$ is still compatible with ${ }^{10} \mathrm{Be} /{ }^{9} \mathrm{Be}$ measurements (e.g., Strong \& Moskalenko 1998).

Considering the large statistical and systematic uncertainties in the SNR distribution, a flatter $\mathrm{CR}$ source distribution in the outer Galaxy also could be possible. We thus tried a modified CR source distribution, in which the distribution is the same as equation 2 below $R_{\mathrm{bk}}$ and constant beyond it (see a thin solid line of Figure 12 as an example). Figure 13 right shows the models with several choices of $R_{\mathrm{bk}}$ for $z_{\mathrm{h}}=4 \mathrm{kpc}$ and $D_{0}=5.8 \times 10^{28} \mathrm{~cm}^{2} \mathrm{~s}^{-1}$. We obtained a reasonable fit to the data using a flat CR source distribution beyond $R=10 \mathrm{kpc}$. Such a constant CR source density in the outer Galaxy is in contrast not only with the (highly uncertain) distribution of SNRs, but also with other tracers of massive star formation and SNRs, like, 1) CO lines which trace the interstellar phase where massive stars form (e.g., Ferrière 2001), 2) OB star counts (e.g., Bronfman et al. 2000), and 3) the ${ }^{26} \mathrm{Al}$ line which is related to the injection of stellar nucleosynthesis products in the ISM by SNRs (Diehl et al. 2006). However, a very large halo size and/or a flat CR source distribution just beyond the solar circle seem to be favored by the LAT data.

The above discussion depends on the propagation parameters and the solution is not unique.
The exploration could be extended to other regions of the parameter space or to a non-uniform diffusion coefficient (e.g., Evoli et al. 2008), but examining propagation models in detail is beyond the scope of our study. Our bottom line is that the analysis of LAT data presented here and by Abdo et al. (2010a) consistently show that the CR density gradient in the outer Galaxy is flatter than expectations by commonly-used propagation models. In the future, the extension to the inner part and the accurate determination of the gradient over the whole Galaxy will be key to constraining the $\mathrm{CR}$ origin and transport.

We also note that a spin temperature $T_{\mathrm{S}} \geq$ $250 \mathrm{~K}$, which is favored by recent studies in the outer Galaxy (e.g., Dickey et al. 2009), gives a small arm/interarm contrast at the 10-20\% level that is not fully compatible with the propagation models (including the one we adopted here) which predict a monotonic CR gradient.

Even though the present analysis includes a dust template to account for the abundant missing gas present locally at the interface between the $\mathrm{HI}$ and CO-bright phases, an alternative way to reconcile the flat emissivity profile and a marked decline in CR density in the outer Galaxy is to invoke an increase in missing gas mass with Galactocentric distance in the low metallicity environments of the outer Galaxy (see e.g. Papadopoulos, Thi \& Viti 2002; Wolfire et al. 2010) beyond the local correction applied here. We note that the large masses of dark gas in the outer Galaxy suggested by Papadopoulos, Thi \& Viti 2002) (outweighing that of $\mathrm{HI}$ by a factor of 5-15) might explain our results, whereas the remarkably constant dark gas fraction of $30 \%$ with mild dependence on metallicity suggested by Wolfire et al. (2010) is not sufficient to explain the large $\mathrm{H}$ i emissivities measured by the LAT beyond the solar circle.

\section{Summary and Conclusion}

We have studied the diffuse $\gamma$-ray emission in the third Galactic quadrant using the first 18 months of Fermi LAT science data. Thanks to the excellent performance of the LAT, we have obtained high-quality emissivity spectra of the atomic and molecular gas (traced by $W_{\mathrm{CO}}$ ) in the $100 \mathrm{MeV}-25.6 \mathrm{GeV}$ energy range. 
At the level of accuracy allowed by the LAT, the study of CR densities from $\gamma$-ray observations is now mostly limited by the understanding of the ISM mass tracers, notably by the uncertainties in the derivation of atomic gas column densities from H I surveys and by the distribution of gas not accounted for by radio and microwave line surveys. In spite of those uncertainties, robust conclusions can be drawn concerning the ISM and CRs.

The molecular mass calibration ratio of the Local arm is found to be $\sim 2 \times 10^{20} \mathrm{~cm}^{-2}\left(\mathrm{~K} \mathrm{~km} \mathrm{~s}^{-1}\right)^{-1}$, significantly larger than that for the very local Gould-Belt clouds in the second Galactic quadrant reported by Abdo et al. (2010a). No significant differences of the ratio are found between the Local arm and the interarm region.

No significant variations in the CR spectra are found across the outer Galaxy in the region studied, and no large contrast in emissivity is seen in the interarm region between the Local and Perseus arms (a contrast $<10-20 \%$ is allowed by data). The measured gradient is much flatter than predictions by a widely-used propagation model assuming that the CR source distribution largely peaks in the inner Galaxy. A larger halo size and/or a flatter $\mathrm{CR}$ source distribution beyond the solar circle than those usually assumed are required to reproduce the LAT data, while other scenarios such as a non-uniform diffusion coefficient or vast amounts of missing gas in the outer Galaxy are also possible. Reliable determinations of the amount of atomic hydrogen in the plane are key to better constraining the property of CRs in our Galaxy.

The Fermi LAT Collaboration acknowledges generous ongoing support from a number of agencies and institutes that have supported both the development and the operation of the LAT as well as scientific data analysis. These include the National Aeronautics and Space Administration and the Department of Energy in the United States, the Commissariat à l'Energie Atomique and the Centre National de la Recherche Scientifique / Institut National de Physique Nucléaire et de Physique des Particules in France, the Agenzia Spaziale Italiana and the Istituto Nazionale di Fisica Nucleare in Italy, the Ministry of Education, Culture, Sports, Science and Technology (MEXT), High Energy Accelerator Research Organization
(KEK) and Japan Aerospace Exploration Agency (JAXA) in Japan, and the K. A. Wallenberg Foundation, the Swedish Research Council and the Swedish National Space Board in Sweden.

Additional support for science analysis during the operations phase is gratefully acknowledged from the Istituto Nazionale di Astrofisica in Italy and the Centre National d'Études Spatiales in France.

This paper makes use of a development version of GALPROP provided by the GALPROP team to the LAT collaboration solely for interpretation of the LAT data. GALPROP development is supported by NASA Grant NNX09AC15G.

We thank T. M. Dame for providing the moment-masked $\mathrm{CO}$ data. 


\section{REFERENCES}

Abdo, A. A. et al. 2009a, ApJ, 703, 1249

Abdo, A. A. et al. 2009b, Phys. Rev. Lett., 102, 181101

Abdo, A. A. et al. 2009c, Astroparticle Physics, 32, 193

Abdo, A. A. et al. 2009d, Science, 323, 1688

Abdo, A. A. et al. 2009e, Nature, 462, 331

Abdo, A. A. et al. 2009f, ApJ, 706L, 138

Abdo, A. A. et al. 2010a, ApJ, 710, 133

Abdo, A. A. et al. 2010b, ApJS, 188, 405

Abdo, A. A. et al. 2010c, ApJS, 187, 460

Abdo, A. A. et al. 2010d, PRL 104, 101101

Abdo, A. A. et al. 2010e, submitted

Atwood, W. B. et al. 2009, ApJ, 697, 1071

IRAS Catalogs and Atlases Explanatory Supplement, 1988, ed. Beichman C., Neugebauer G., Habing H. J., Clegg P. E., and Chester T. J. (Washington, DC: GPO), NASA RP-1190, vol 1

Bloemen, J. B. G. et al. 1986, A\&A, 154, 25

Bloemen, H. 1989, Annu. Rev. Astron. Astrophys., 27,469

Bronfman, L. et al. 2000, A\&A, 358, 521

Case, G. L., \& Bhattacharya, D. 1998, ApJ, 504, 761

Cordes, J. M., \& Lazio, T. J. W. 2002, arXiv:astro-ph/0207156

Clemens, D. P. 1985, ApJ, 295, 422

Dame, T. M., Hartmann, D., \& Thaddeus, P. 2001, ApJ, 547, 792

Dickey, J. M. et al. 2009, ApJ, 693, 1250

Diehl, R. et al. 2006, Nature, 439, 45

Digel, S. W., Grenier, I. A., Hunter, S. D., Dame, T. M., \& Thaddeus, P. 2001, ApJ, 555, 12
Elad, M. et al. 2005, Applied and Computational Harmonic Analysis, 19, 340

Evoli, C., Gaggero, D., Grasso, D., Maccione, L. 2008, Journal of Cosmology and Astroparticle Physics, 10, 18

Ferrière, K. M. 2001, Reviews of Modern Physics, 73,1031

Gibson, S. J., Taylor, A. R., Higgs, L. A., Brunt, C. M., \& Dewdney, P. E. 2005, ApJ, 626, 195

Grenier, I. A., Casandjian, J.-M., \& Terrier, R. 2005, Science, 307, 1292

Henderson, A. P., Jackson, P. D., \& Kerr, F. J. 1982, ApJ, 263, 116

Hunter, S. D., Digel, S. W., de Geus, E. J. \& Kanbach, G. 1994, ApJ, 436, 216

Hunter, S. D., et al. 1997, ApJ, 481, 205

Kalberla, P. M. W., Burton, W. B., Hartmann, D., Arnal, E. M., Bajaja, E., Morras R., Pöppel, W. G. L., 2005, A\&A, 440, 775

Kaltcheva, N. T. \& Hilditch, R. W. 2000, MNRAS, 312,753

Lebrun, F. et al. 1983, ApJ, 274, 231

Lorimer, D. R. 2004, arXiv:astro-ph/0308501

Maddalena, R. J., \& Thaddeus, P. 1985, ApJ, 294, 231

Maddalena, R. J., Morris, M., Moscowitz, J., \& Thaddeus, P. 1986, ApJ, 303, 375

Mattox, J. R. et al. 1996, ApJ, 461, 396

Mel'nik, A. M., \& Efremov, Yu. N. 1995, Astronomy Letters, 21, 10

Mori, M. 1997, ApJ, 478, 225

Morris, M., Montani, J., \& Thaddeus, P. 1979, IAUS, 87, 197

Papadopoulos, P. P., Thi, W. -F., \& Viti, S. 2002, ApJ, 579, 270

Porter, T. A., Moskalenko, I. V., Strong, A. W., Orlando, E. and Bouchet, L. 2008, ApJ, 682, 400 
Rando, R. et al. 2009, arXiv:0907.0626

Schlegel, D. J., Finkbeiner, D. P., \& Daris, M. 1998, ApJ, 500, 525

Stecker, F. W., \& Jones, F. C. 1977, ApJ, 217, 843

Strong, A. W., et al. 1988, A\&A, 207, 1

Strong, A. W., \& Mattox, J. R. 1996, A\&A, 308, L21

Strong, A. W., \& Moskalenko, I. V. 1998, ApJ, 509,212

Strong, A. W., Moskalenko, I. V., \& Reimer, O. 2004a, ApJ, 613, 962

Strong, A. W., Moskalenko, I. V., Reimer, O., Digel, S., \& Diehl, R. 2004b, A\&A 2004, 422, L47

Strong, A. W., Moskalenko, I. V., \& Ptuskin, V. S. 2007, Annual Review of Nuclear and Particle Systems, 57, 1, 285

Wilson, B. A., Dame, T. M., Masheder, M. R. W., \& Thaddeus, P. 2005, ApJ, 430, 523

Wolfire, M. G., Hollenbach, D., \& McKee, C. F. 2010, ApJ, 716, 1191

This 2-column preprint was prepared with the AAS IATEX macros v5.2. 

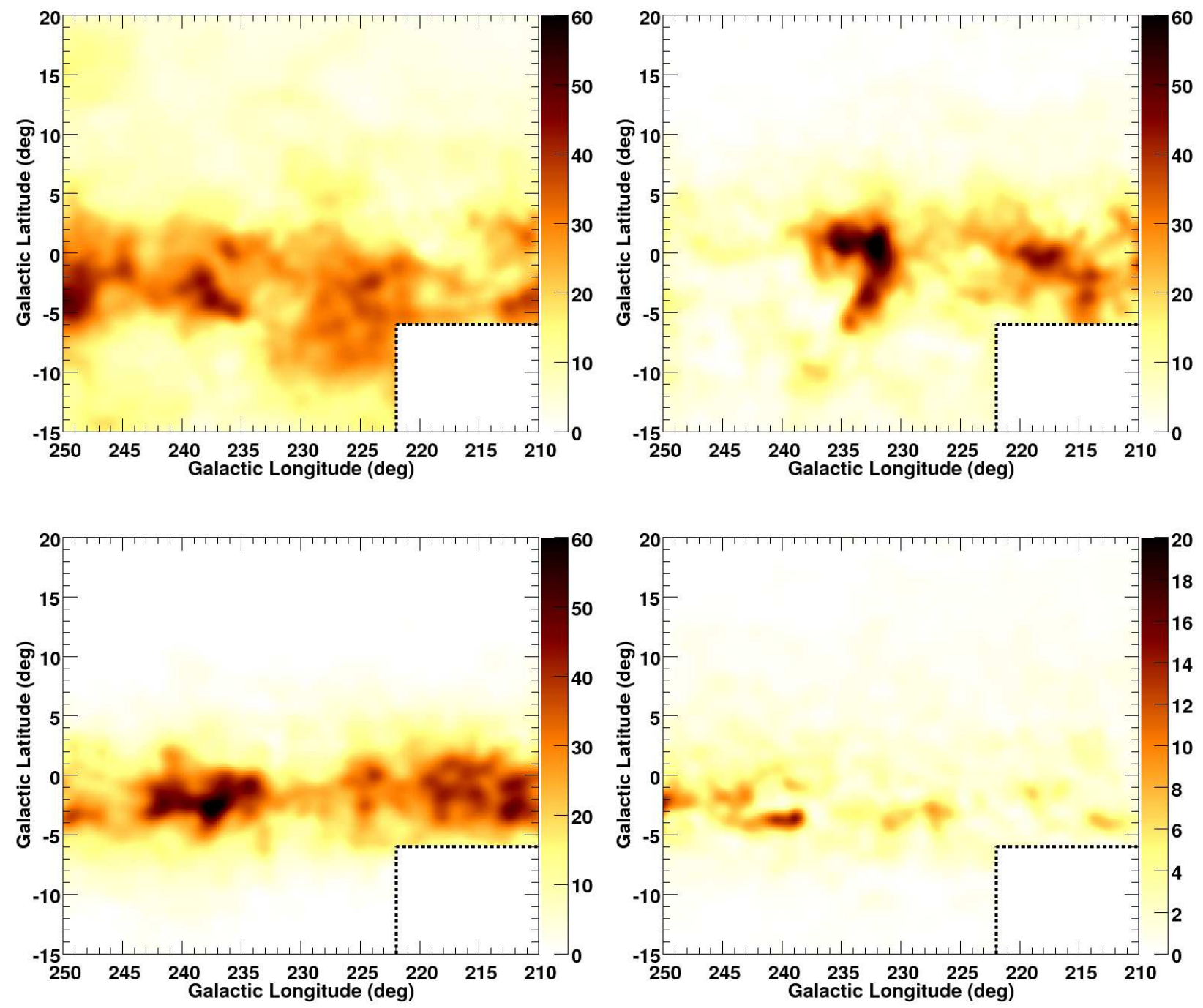

Fig. 2.- Maps of $N(\mathrm{HI})$ (in unit of $10^{20}$ atoms $\mathrm{cm}^{-2}$ ) for the Local arm (top left), interarm (top right), Perseus arm (bottom left), and outer arm (bottom right) regions, obtained for a spin temperature $T_{\mathrm{S}}=125 \mathrm{~K}$. The outlined area in the bottom right corner is not used in the analysis (see $\S 1$ ). The maps have been smoothed with a Gaussian with $\sigma=1^{\circ}$ for display. 

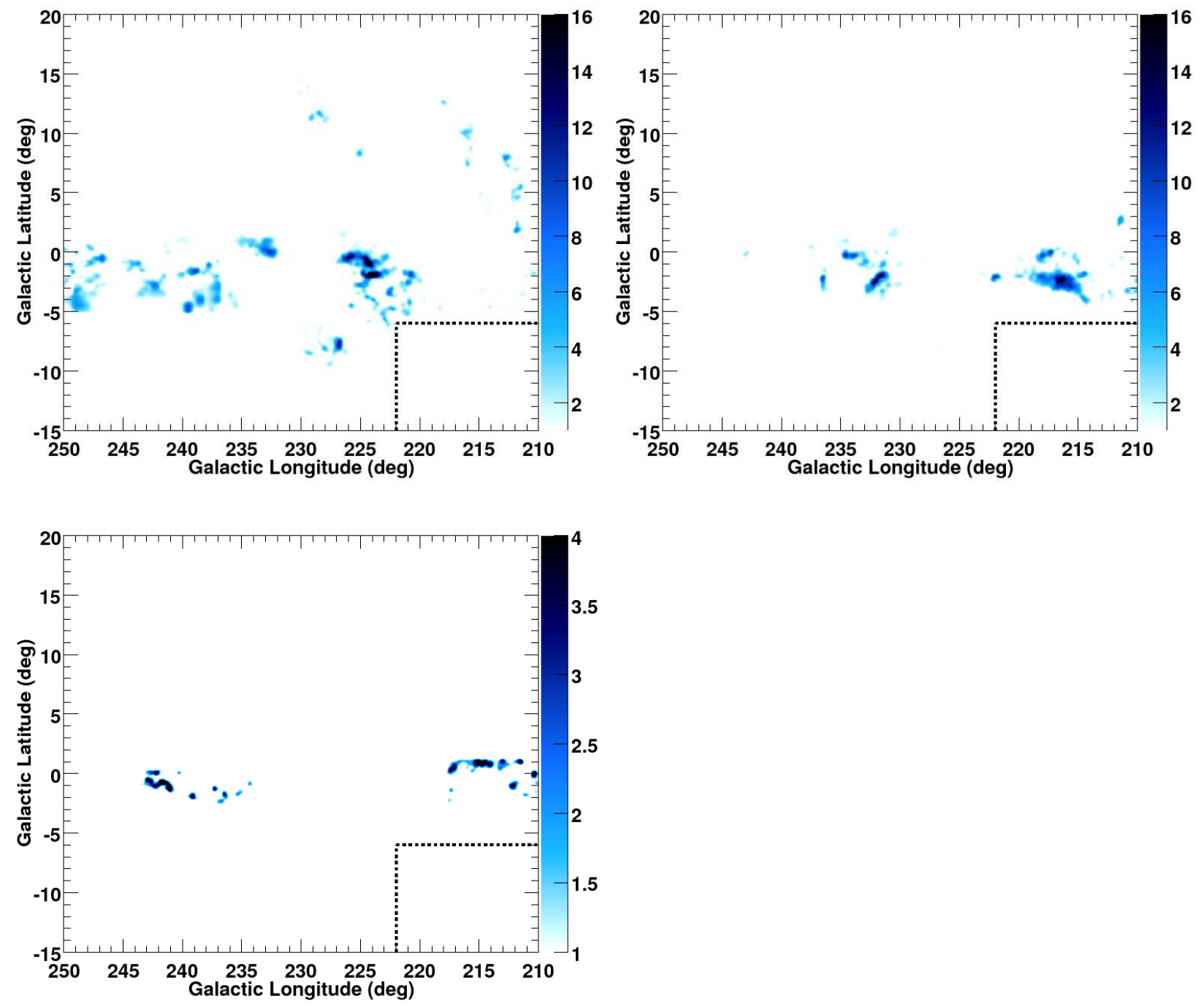

Fig. 3.- Maps of $W_{\mathrm{CO}}$ (in unit of $\mathrm{K} \mathrm{km} \mathrm{s}^{-1}$ ) for the Local arm (top left), interarm (top right), and the Perseus arm (bottom left) regions. The small box in the bottom right corner indicates the area not considered in the analysis. The maps have been smoothed with a Gaussian of $\sigma=0.25^{\circ}$ for display. 

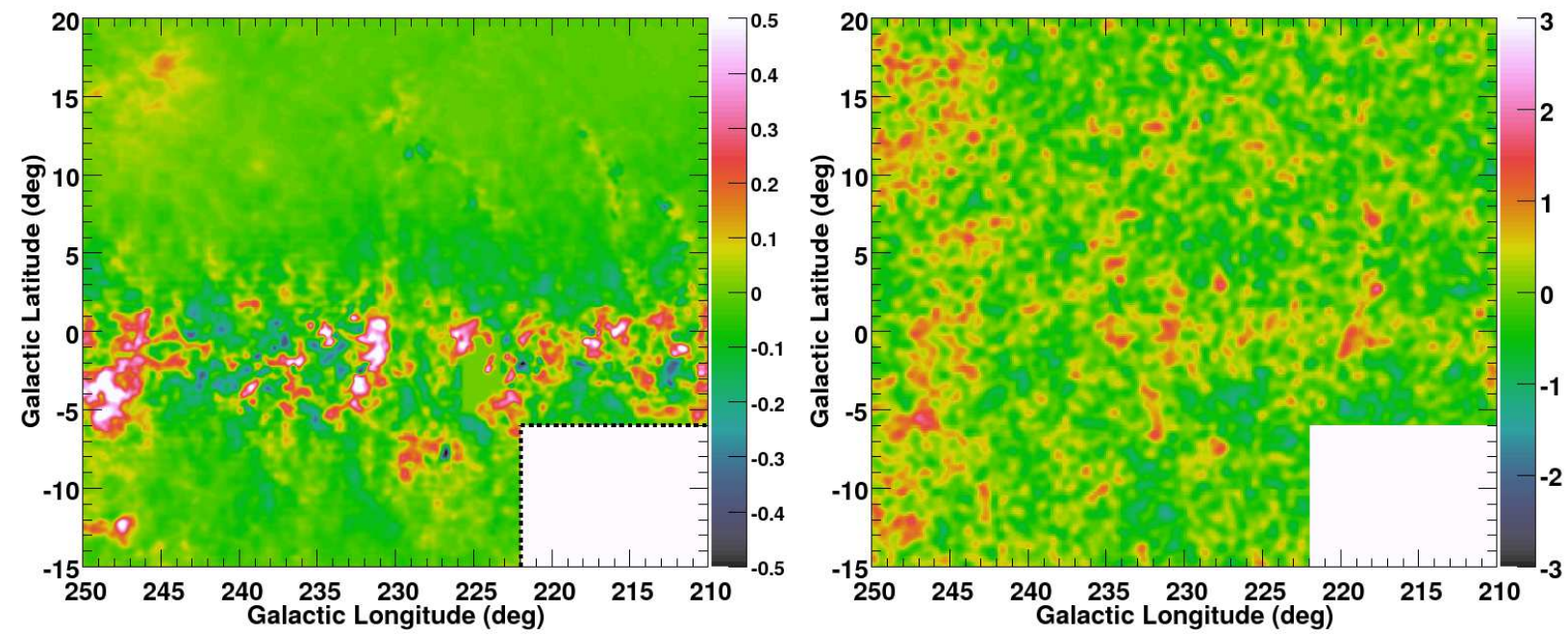

Fig. 4.- (left) Residual $E(B-V)$ map in unit of magnitudes, obtained by subtracting the parts linearly correlated with the combination of $N(\mathrm{HI})$ and $W_{\mathrm{CO}}$ maps. The small box in the bottom right corner shows the area not considered in the analysis. The map has been smoothed with a Gaussian of $\sigma=0.25^{\circ}$ for display. (right) $\gamma$-ray residual (data minus model) map obtained by the fit without the $E(B-V)_{\text {res }}$ map (only $\mathrm{H}$ I and $\mathrm{CO}$ maps) in unit of standard deviations (square root of model-predicted counts, saturated between $-3 \sigma$ and $+3 \sigma$ ). The map has been smoothed with a Gaussian of $\sigma=0.5^{\circ}$. 

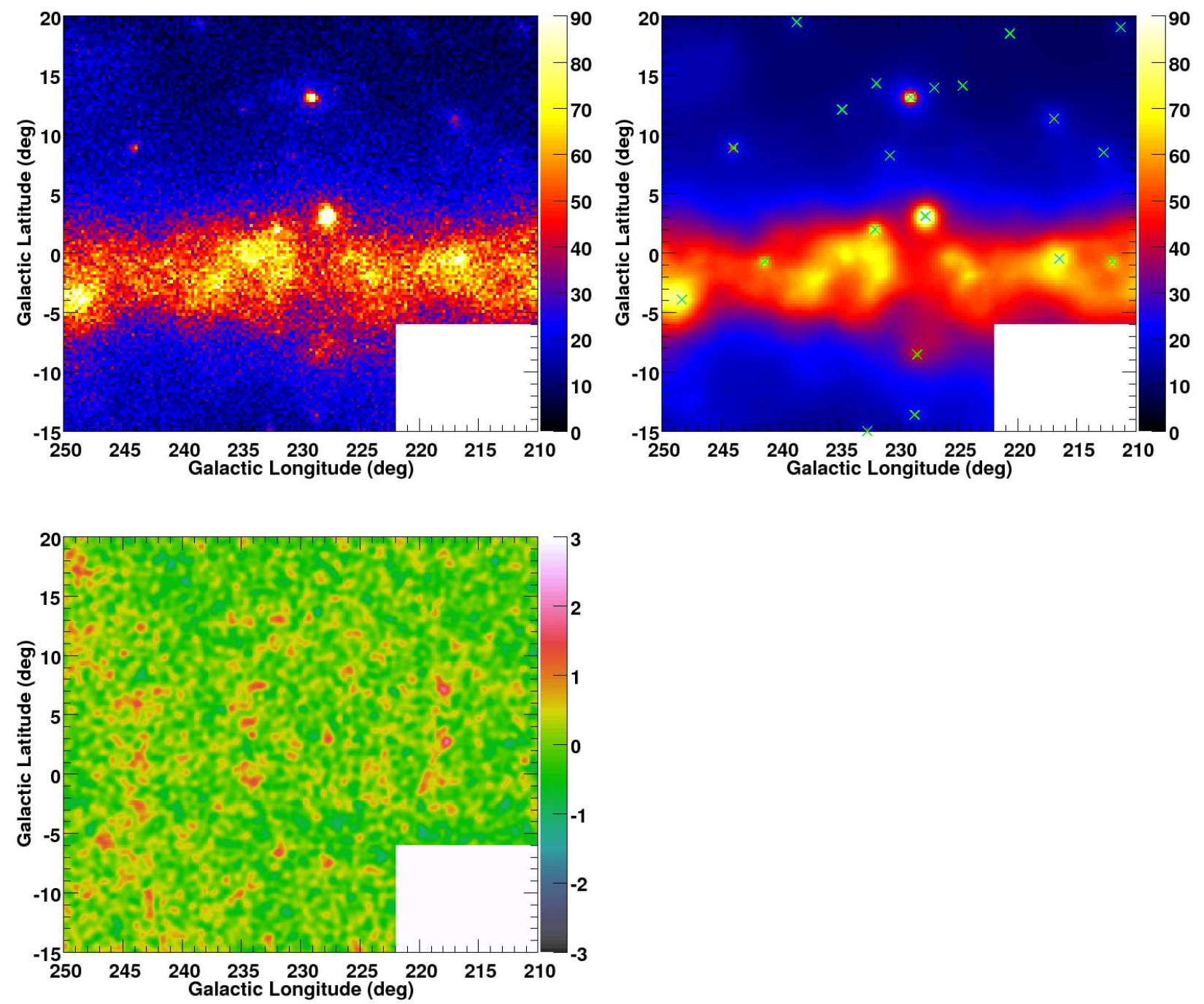

Fig. 5.- Data count map (top left), model count map (top right) and the residual (data minus model) map in units of standard deviations (bottom left, saturated between $-3 \sigma$ and $+3 \sigma$ ) above $100 \mathrm{MeV}$ obtained by our analysis. Point sources with TS $\geq 50$ in the 1FGL included in the fit are shown by crosses in the model map. Data/model count maps are in $0.25^{\circ} \times 0.25^{\circ}$ pixels, and the residual map has been smoothed with a Gaussian of $\sigma=0.5^{\circ}$. 


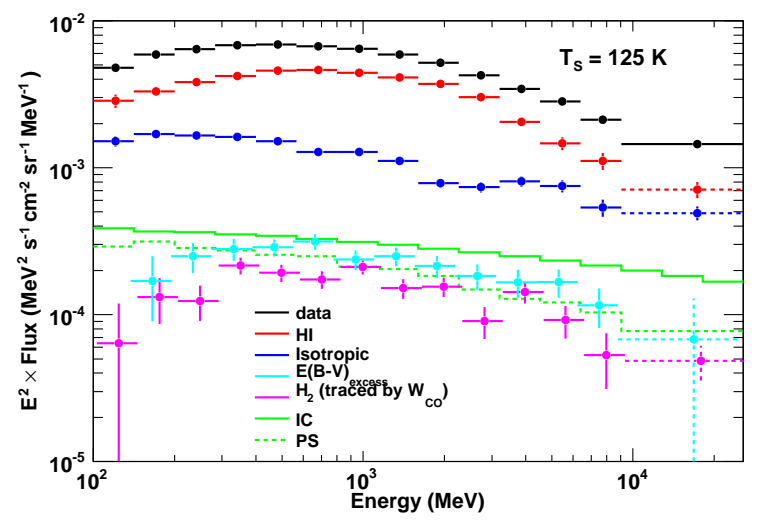

Fig. 6. - $\gamma$-ray spectra over the region of interest obtained from data and from the fitted model (for each gas phase, IC and isotropic components, and for point sources).

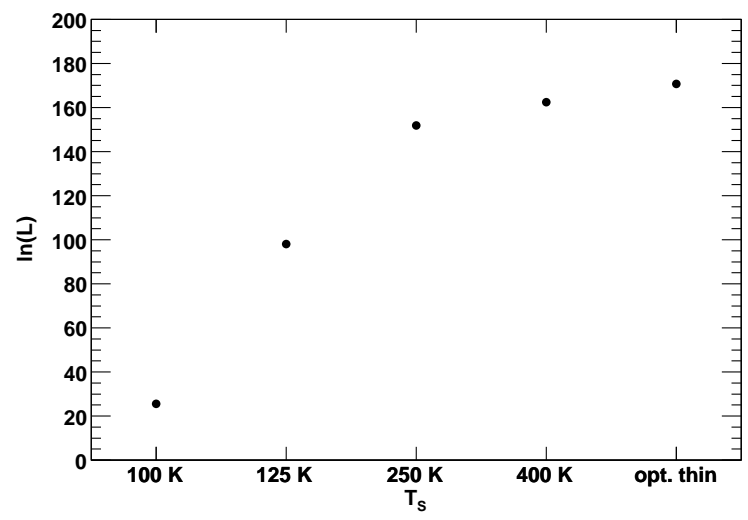

Fig. 7.- Variations of the log-likelihood value for several choices of $T_{\mathrm{S}}$ (the scale has a fixed offset). 

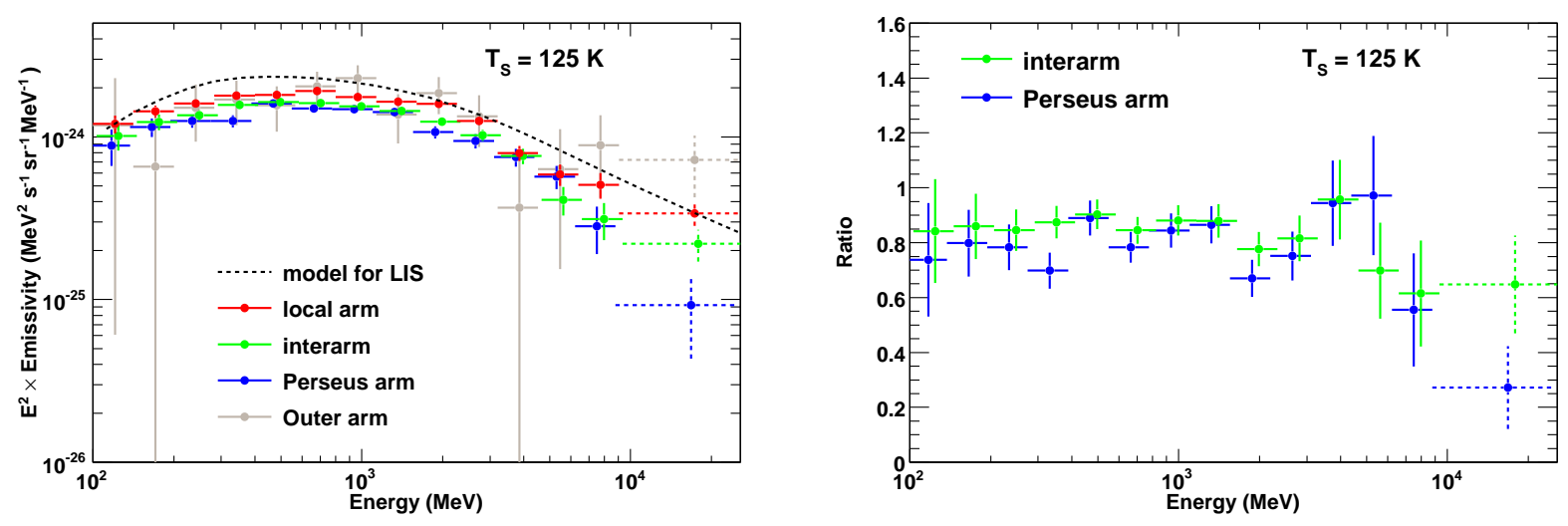

Fig. 8. - (left) H I emissivity spectra obtained for each region. For reference, the emissivity model spectrum for the LIS adopted by Abdo et al. (2009a) is shown by the black dotted line. (right) The emissivity ratios to that of the Local arm. In both panels, $T_{\mathrm{S}}=125 \mathrm{~K}$ is assumed and spectra in the interarm region and the Perseus arm are shifted horizontally for clarity.
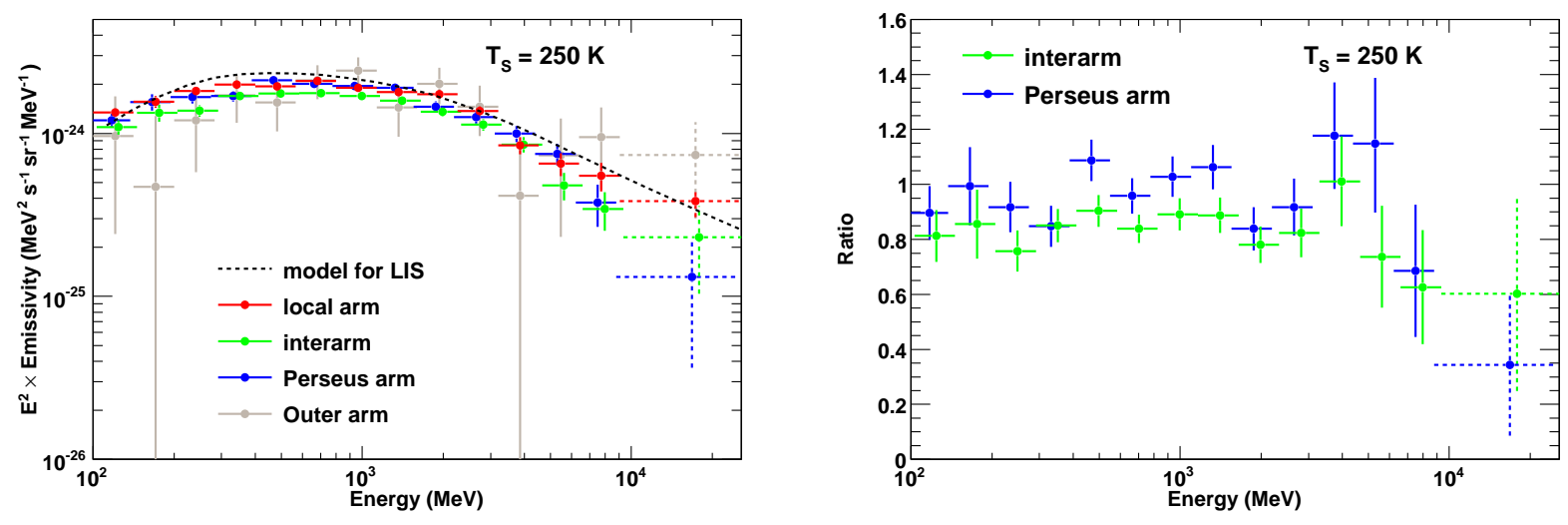

Fig. 9. - The same as Figure 8 but for $T_{\mathrm{S}}=250 \mathrm{~K}$. 

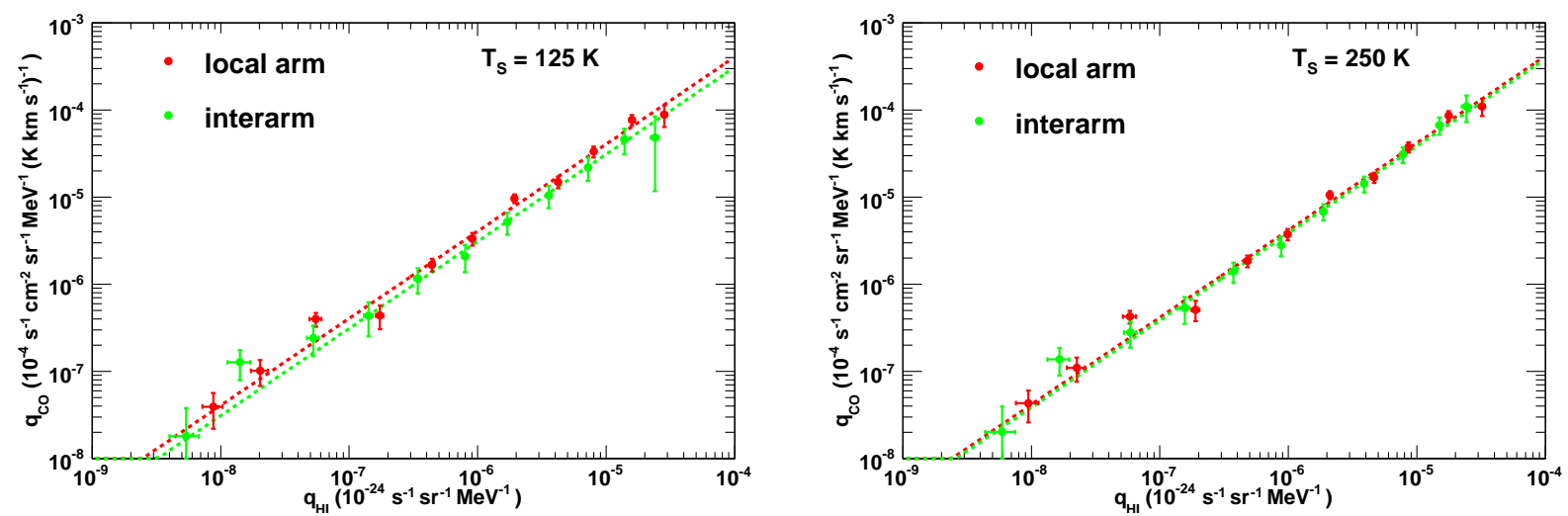

Fig. 10.- Correlation between the $\mathrm{HI}$ and $\mathrm{CO}$ emissivities in the $200 \mathrm{MeV}-9.05 \mathrm{GeV}$ energy range for the Local arm and the interarm regions. The cases of $T_{\mathrm{S}}=125 \mathrm{~K}$ and $250 \mathrm{~K}$ are shown in the left panel and the right panel, respectively. Dotted lines show the best linear fits. Each data point corresponds to an energy bin used in the $\gamma$-ray analysis. (See Table 1 and 2)
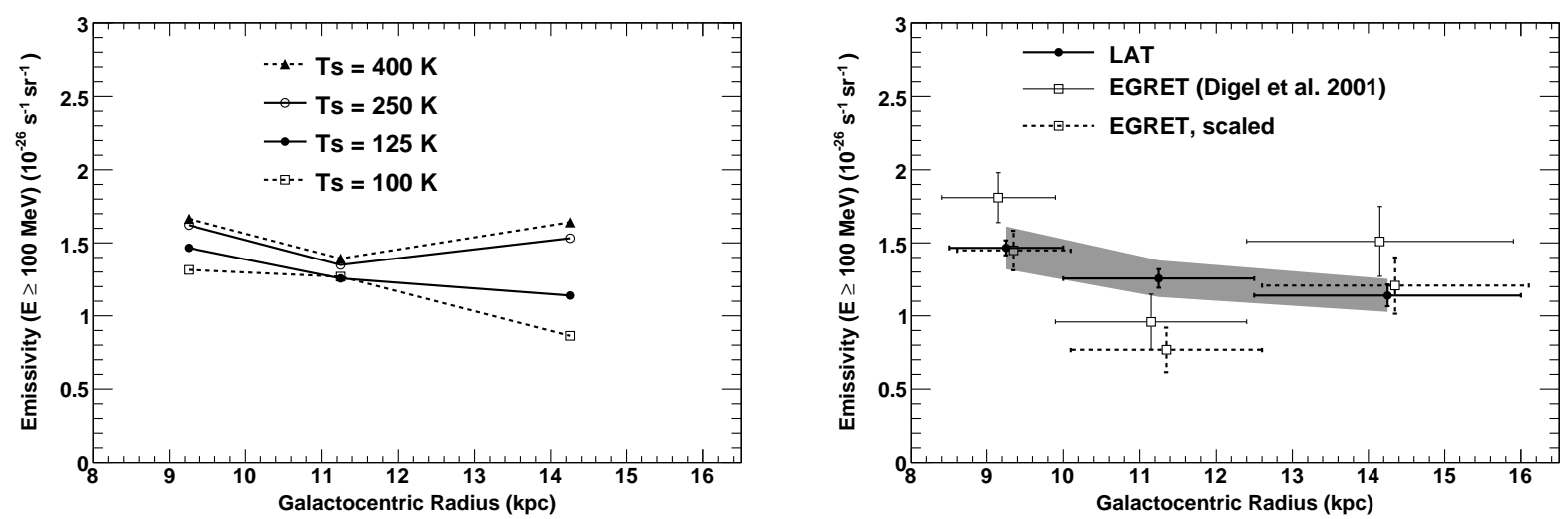

Fig. 11. - (left) Emissivity gradient for several choices of $T_{\mathrm{S}}$. (right) Emissivity gradient obtained by the LAT compared with the EGRET results under the assumption of $T_{\mathrm{S}}=125 \mathrm{~K}$. The shaded area indicates the systematic uncertainty in the LAT selection efficiency of $\sim 10 \%$. The EGRET points have been downscaled by $20 \%$ to account for the change in H I survey data between the two studies (see $\S$ 4.3.1). 


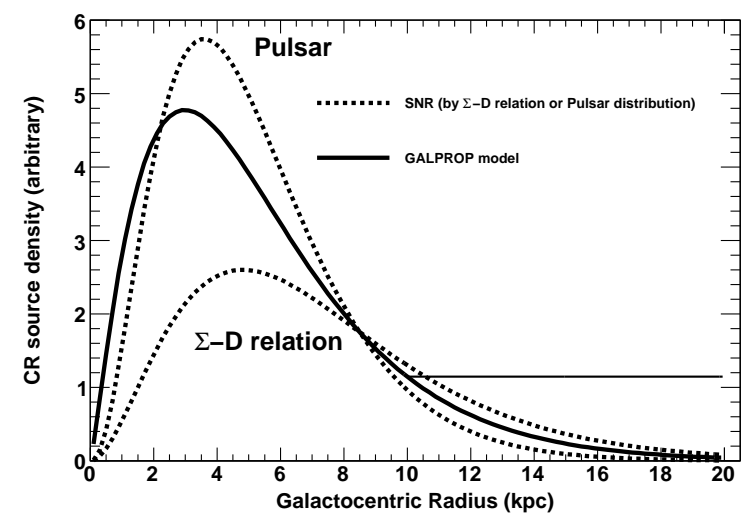

Fig. 12.- The CR source distribution adopted in our baseline GALPROP model (solid line), compared with the SNR distribution obtained by the $\Sigma-D$ relation (Case \& Bhattacharya 1998) and that traced by the pulsar distribution (Lorimer 2004) shown by dotted lines. The thin solid line represents an example of the modified distributions introduced to reproduce the emissivity gradient by the LAT.
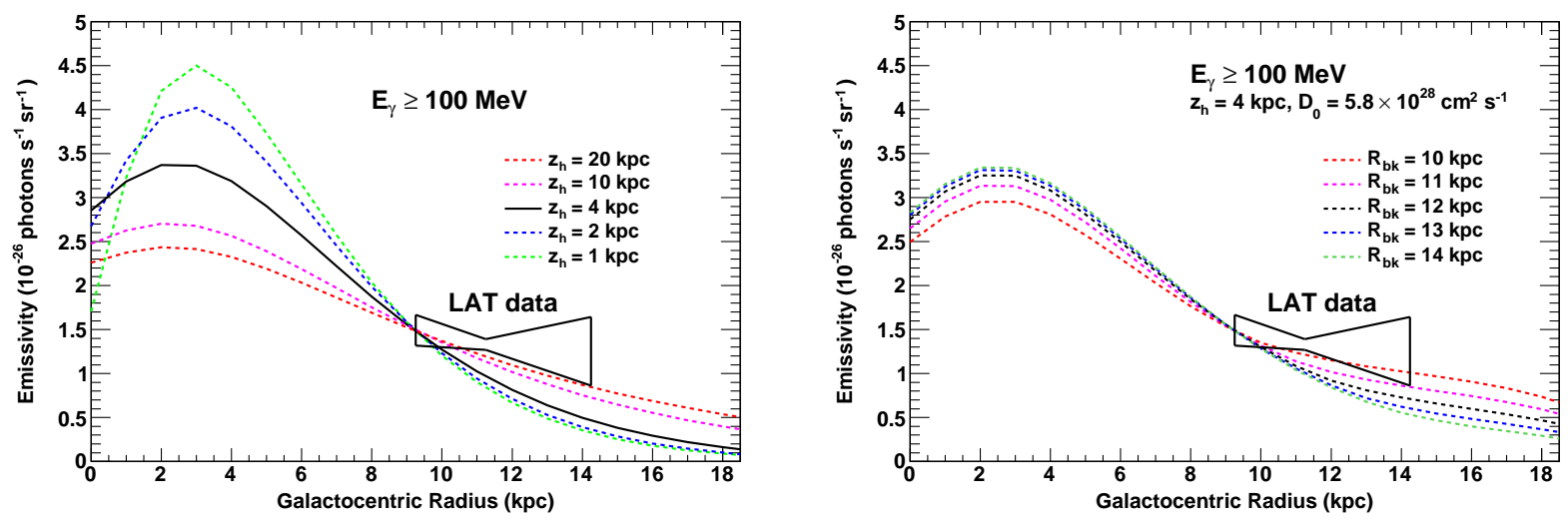

Fig. 13. - Comparison of the emissivity gradient obtained by the LAT and model expectations using GALPROP. The left panel shows models with different halo sizes and diffusion lengths: $\left(z_{\mathrm{h}}, D_{0}\right)=(1 \mathrm{kpc}$, $\left.1.7 \times 10^{28} \mathrm{~cm}^{2} \mathrm{~s}^{-1}\right),\left(2 \mathrm{kpc}, 3.2 \times 10^{28} \mathrm{~cm}^{2} \mathrm{~s}^{-1}\right),\left(4 \mathrm{kpc}, 5.8 \times 10^{28} \mathrm{~cm}^{2} \mathrm{~s}^{-1}\right),\left(10 \mathrm{kpc}, 12 \times 10^{28} \mathrm{~cm}^{2} \mathrm{~s}^{-1}\right)$ and $\left(20 \mathrm{kpc}, 18 \times 10^{28} \mathrm{~cm}^{2} \mathrm{~s}^{-1}\right)$. The solid line is for $z_{\mathrm{h}}=4 \mathrm{kpc}$. The right panel shows different choices of the break distance beyond which a flat CR source distribution is assumed: $R_{\mathrm{bk}}=10-14 \mathrm{kpc}$ with $1 \mathrm{kpc}$ steps. 
TABLE 1

A SUMmaRY OF FIT PARAMETERS WITH 1 SIgMa STATISTICAL ERRORS, UNDER THE ASSUMPTION OF $T_{\mathrm{S}}=125 \mathrm{~K}$.

\begin{tabular}{|c|c|c|c|c|c|c|c|c|c|c|}
\hline $\begin{array}{c}\text { Energy } \\
\text { GeV }\end{array}$ & $E^{2} \cdot q_{\mathrm{HI}, 1}$ & $E^{2} \cdot q_{\mathrm{HI}, 2}$ & $E^{2} \cdot q_{\mathrm{HI}, 3}$ & $E^{2} \cdot q_{\mathrm{HI}, 4}$ & $E^{2} \cdot q_{\mathrm{CO}, 1}$ & $E^{2} \cdot q_{\mathrm{CO}, 2}$ & $E^{2} \cdot q_{\mathrm{CO}, 3^{\mathrm{c}}}$ & $E^{2} \cdot q_{\mathrm{EBV}_{\mathrm{pos}}}$ & $\overline{E^{2} \cdot q_{\mathrm{EBV}}}$ neg & $E^{2} \cdot q_{\text {iso }}$ \\
\hline $0.10-0.14$ & $1.19 \pm 0.15$ & $1.01 \pm 0.18$ & $0.88 \pm 0.22$ & $1.1 \pm 1.1$ & $0.0 \pm 0.1$ & $7 \pm 6$ & $6 \pm 24$ & $0.00 \pm 0.08$ & $1.04 \pm 0.45$ & $1.73 \pm 0.14$ \\
\hline $0.14-0.20$ & $1.43 \pm 0.11$ & $1.23 \pm 0.13$ & $1.14 \pm 0.14$ & $0.7 \pm 0.8$ & $4.3 \pm 1.8$ & $5.0 \pm 2.9$ & $0 \pm 1$ & $0.77 \pm 0.36$ & $0.35 \pm 0.34$ & $2.01 \pm 0.09$ \\
\hline $0.20-0.28$ & $1.60 \pm 0.08$ & $1.36 \pm 0.10$ & $1.25 \pm 0.11$ & $1.5 \pm 0.6$ & $5.0 \pm 1.3$ & $2.7 \pm 2.0$ & $0 \pm 1$ & $1.13 \pm 0.26$ & $0.32 \pm 0.24$ & $1.94 \pm 0.07$ \\
\hline $0.28-0.40$ & $1.79 \pm 0.07$ & $1.57 \pm 0.08$ & $1.25 \pm 0.10$ & $1.7 \pm 0.5$ & $8.6 \pm 1.2$ & $5.2 \pm 1.6$ & $11 \pm 7$ & $1.28 \pm 0.21$ & $0.45 \pm 0.20$ & $1.90 \pm 0.06$ \\
\hline $0.40-0.56$ & $1.81 \pm 0.07$ & $1.63 \pm 0.08$ & $1.61 \pm 0.10$ & $1.6 \pm 0.5$ & $7.5 \pm 1.1$ & $4.9 \pm 1.4$ & $8 \pm 6$ & $1.30 \pm 0.17$ & $0.87 \pm 0.18$ & $1.74 \pm 0.06$ \\
\hline $0.56-0.80$ & $1.91 \pm 0.07$ & $1.62 \pm 0.07$ & $1.50 \pm 0.09$ & $2.0 \pm 0.5$ & $6.7 \pm 1.0$ & $4.7 \pm 1.3$ & $3 \pm 5$ & $1.43 \pm 0.17$ & $0.88 \pm 0.17$ & $1.49 \pm 0.06$ \\
\hline $0.80-1.13$ & $1.75 \pm 0.07$ & $1.54 \pm 0.07$ & $1.48 \pm 0.09$ & $2.3 \pm 0.5$ & $8.7 \pm 1.0$ & $4.6 \pm 1.3$ & $16 \pm 5$ & $1.07 \pm 0.16$ & $0.88 \pm 0.16$ & $1.48 \pm 0.06$ \\
\hline $1.13-1.60$ & $1.64 \pm 0.07$ & $1.45 \pm 0.08$ & $1.42 \pm 0.09$ & $1.4 \pm 0.5$ & $6.0 \pm 1.0$ & $3.7 \pm 1.3$ & $11 \pm 5$ & $1.13 \pm 0.16$ & $0.76 \pm 0.16$ & $1.28 \pm 0.06$ \\
\hline $1.60-2.26$ & $1.60 \pm 0.08$ & $1.24 \pm 0.08$ & $1.07 \pm 0.09$ & $1.9 \pm 0.5$ & $6.0 \pm 1.0$ & $4.1 \pm 1.3$ & $7 \pm 4$ & $0.97 \pm 0.16$ & $0.53 \pm 0.16$ & $0.91 \pm 0.07$ \\
\hline $2.26-3.20$ & $1.26 \pm 0.08$ & $1.02 \pm 0.08$ & $0.94 \pm 0.09$ & $1.3 \pm 0.5$ & $3.2 \pm 1.0$ & $3.1 \pm 1.3$ & $3 \pm 4$ & $0.82 \pm 0.16$ & $0.58 \pm 0.16$ & $0.85 \pm 0.07$ \\
\hline $3.20-4.53$ & $0.80 \pm 0.09$ & $0.76 \pm 0.08$ & $0.75 \pm 0.09$ & $0.4 \pm 0.5$ & $5.7 \pm 1.0$ & $3.5 \pm 1.3$ & $11 \pm 4$ & $0.74 \pm 0.16$ & $0.42 \pm 0.16$ & $0.93 \pm 0.08$ \\
\hline $4.53-6.40$ & $0.59 \pm 0.09$ & $0.41 \pm 0.08$ & $0.57 \pm 0.09$ & $0.6 \pm 0.5$ & $2.9 \pm 1.0$ & $3.6 \pm 1.3$ & $0 \pm 0$ & $0.74 \pm 0.16$ & $0.02 \pm 0.17$ & $0.86 \pm 0.08$ \\
\hline $6.40-9.05$ & $0.51 \pm 0.09$ & $0.31 \pm 0.08$ & $0.28 \pm 0.09$ & $0.9 \pm 0.5$ & $2.2 \pm 1.0$ & $1.0 \pm 1.1$ & $2 \pm 3$ & $0.52 \pm 0.15$ & $0.07 \pm 0.16$ & $0.62 \pm 0.08$ \\
\hline $9.05-25.6$ & $0.34 \pm 0.05$ & $0.22 \pm 0.05$ & $0.09 \pm 0.05$ & $0.7 \pm 0.3$ & $2.2 \pm 0.6$ & $1.4 \pm 0.7$ & $3 \pm 2$ & $0.07 \pm 0.06$ & $0.06 \pm 0.08$ & $0.49 \pm 0.05$ \\
\hline
\end{tabular}

a Units: $\quad E^{2} \cdot q_{\mathrm{HI}, \mathrm{i}}\left(10^{-24} \mathrm{MeV}^{2} \mathrm{~s}^{-1} \mathrm{sr}^{-1} \mathrm{MeV}^{-1}\right), \quad E^{2} \quad q_{\mathrm{CO}, \mathrm{i}}\left(10^{-4} \mathrm{MeV}^{2} \mathrm{~s}^{-1} \mathrm{~cm}^{-2} \mathrm{sr}^{-1} \mathrm{MeV}^{-1}\left(\mathrm{~K} \mathrm{~km} \mathrm{~s}^{-1}\right)^{-1}\right), \quad E^{2} \quad$. $q_{\mathrm{EBV}}\left(10^{-2} \mathrm{MeV}^{2} \mathrm{~s}^{-1} \mathrm{~cm}^{-2} \mathrm{sr}^{-1} \mathrm{MeV}^{-1} \mathrm{mag}^{-1}\right), E^{2} \cdot q_{\mathrm{iso}}\left(10^{-3} \mathrm{MeV}^{2} \mathrm{~s}^{-1} \mathrm{~cm}^{-2} \mathrm{sr}^{-1} \mathrm{MeV}^{-1}\right)$

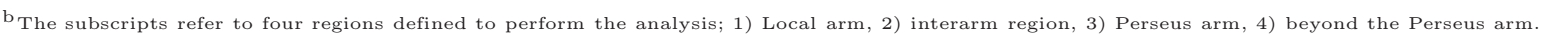

${ }^{\mathrm{c}}$ Some parameters are not well determined and their best-fit value is consistent with 0 . We present them for completeness.

TABLE 2

A SUMMARY OF FIT PARAMETERS WITH 1 SIGMA STATISTICAL ERRORS, UNDER THE ASSUMPTION OF $T_{\mathrm{S}}=250 \mathrm{~K}$.

\begin{tabular}{|c|c|c|c|c|c|c|c|c|c|}
\hline $\begin{array}{c}\text { Energy } \\
\text { GeV }\end{array}$ & $E^{2} \cdot q_{\mathrm{HI}, 1}$ & $E^{2} \cdot q_{\mathrm{HI}, 2}$ & $E^{2} \cdot q_{\mathrm{HI}, 3}$ & $E^{2} \cdot q_{\mathrm{HI}, 4}$ & $E^{2} \cdot q_{\mathrm{CO}, 1}$ & $E^{2} \cdot q_{\mathrm{CO}, 2}$ & $E^{2} \cdot q_{\mathrm{CO}, 3^{\mathrm{c}}}$ & $E^{2} \cdot q_{\mathrm{EBV}}$ pos & $E^{2} \cdot q_{\mathrm{EBV}}$ neg \\
\hline $0.10-0.14$ & $1.35 \pm 0.07$ & $1.09 \pm 0.11$ & $1.20 \pm 0.11$ & $1.0 \pm 0.7$ & $0.1 \pm 0.7$ & $9 \pm 6$ & $7 \pm 66$ & $0.00 \pm 0.03$ & $0.96 \pm 0.18$ \\
\hline $0.14-0.20$ & $1.56 \pm 0.13$ & $1.33 \pm 0.15$ & $1.55 \pm 0.17$ & $0.5 \pm 0.8$ & $5.2 \pm 1.8$ & $7.1 \pm 3.0$ & $0 \pm 1$ & $0.79 \pm 0.33$ & $0.44 \pm 0.29$ \\
\hline $0.20-0.28$ & $1.82 \pm 0.10$ & $1.37 \pm 0.11$ & $1.66 \pm 0.13$ & $1.2 \pm 0.6$ & $6.2 \pm 1.3$ & $6.1 \pm 2.0$ & $0 \pm 1$ & $1.11 \pm 0.23$ & $0.24 \pm 0.20$ \\
\hline $0.28-0.40$ & $2.00 \pm 0.09$ & $1.70 \pm 0.09$ & $1.69 \pm 0.12$ & $1.7 \pm 0.6$ & $9.7 \pm 1.2$ & $7.5 \pm 1.6$ & $13 \pm 8$ & $1.19 \pm 0.19$ & $0.55 \pm 0.17$ \\
\hline $0.40-0.56$ & $1.95 \pm 0.08$ & $1.76 \pm 0.09$ & $2.11 \pm 0.11$ & $1.5 \pm 0.5$ & $8.4 \pm 1.1$ & $7.0 \pm 1.4$ & $9 \pm 6$ & $1.33 \pm 0.14$ & $0.83 \pm 0.14$ \\
\hline $0.56-0.80$ & $2.10 \pm 0.08$ & $1.76 \pm 0.08$ & $2.01 \pm 0.11$ & $2.1 \pm 0.5$ & $7.6 \pm 1.0$ & $6.4 \pm 1.3$ & $4 \pm 5$ & $1.33 \pm 0.15$ & $0.92 \pm 0.14$ \\
\hline $0.80-1.13$ & $1.90 \pm 0.08$ & $1.70 \pm 0.08$ & $1.95 \pm 0.10$ & $2.4 \pm 0.5$ & $9.6 \pm 1.0$ & $6.1 \pm 1.3$ & $17 \pm 5$ & $1.03 \pm 0.14$ & $0.88 \pm 0.14$ \\
\hline $1.13-1.60$ & $1.79 \pm 0.09$ & $1.59 \pm 0.09$ & $1.90 \pm 0.10$ & $1.4 \pm 0.5$ & $6.7 \pm 1.0$ & $5.0 \pm 1.3$ & $11 \pm 5$ & $1.05 \pm 0.14$ & $0.79 \pm 0.14$ \\
\hline $1.60-2.26$ & $1.74 \pm 0.09$ & $1.36 \pm 0.09$ & $1.45 \pm 0.11$ & $2.0 \pm 0.5$ & $6.6 \pm 1.0$ & $5.0 \pm 1.3$ & $7 \pm 4$ & $0.98 \pm 0.15$ & $0.57 \pm 0.14$ \\
\hline $3.20-4.53$ & $0.84 \pm 0.10$ & $0.85 \pm 0.09$ & $0.99 \pm 0.11$ & $0.4 \pm 0.5$ & $6.1 \pm 1.0$ & $4.0 \pm 1.3$ & $11 \pm 4$ & $0.71 \pm 0.14$ & $0.43 \pm 0.14$ \\
\hline $4.53-6.40$ & $0.65 \pm 0.10$ & $0.48 \pm 0.09$ & $0.74 \pm 0.11$ & $0.7 \pm 0.5$ & $3.2 \pm 1.0$ & $3.9 \pm 1.3$ & $0 \pm 0$ & $0.65 \pm 0.15$ & $0.13 \pm 0.15$ \\
\hline $6.40-9.05$ & $0.54 \pm 0.10$ & $0.34 \pm 0.09$ & $0.37 \pm 0.10$ & $0.9 \pm 0.5$ & $2.5 \pm 1.0$ & $1.1 \pm 1.1$ & $2 \pm 3$ & $0.55 \pm 0.14$ & $0.06 \pm 0.15$ \\
\hline $9.05-25.6$ & $0.38 \pm 0.08$ & $0.23 \pm 0.13$ & $0.13 \pm 0.10$ & $0.7 \pm 0.4$ & $2.3 \pm 0.6$ & $1.5 \pm 0.7$ & $3 \pm 2$ & $0.1 \pm 0.3$ & $4 \pm 1$ \\
\hline
\end{tabular}

$E^{2} \cdot q_{\text {iso }}$

$1.68 \pm 0.07$ $1.97 \pm 0.10$ $1.83 \pm 0.07$
$1.83 \pm 0.06$ $1.68 \pm 0.06$ $1.42 \pm 0.06$
$1.41 \pm 0.06$ $1.41 \pm 0.06$ $0.85 \pm 0.07$ $0.85 \pm 0.07$
$0.79 \pm 0.08$ $0.91 \pm 0.08$ $0.84 \pm 0.09$ $0.84 \pm 0.09$
$0.59 \pm 0.09$ $0.45 \pm 0.14$ $q_{\mathrm{EBV}}\left(10^{-2} \mathrm{MeV}^{2} \mathrm{~s}^{-1} \mathrm{~cm}^{-2} \mathrm{sr}^{-1} \mathrm{MeV}^{-1} \mathrm{mag}^{-1}\right), \mathrm{E}^{2} \cdot q_{\mathrm{iso}}\left(10^{-3} \mathrm{MeV}^{2} \mathrm{~s}^{-1} \mathrm{~cm}^{-2} \mathrm{sr}^{-1} \mathrm{MeV}^{-1}\right)$

b The subscripts refer to four regions defined to perform the analysis; 1) Local arm, 2) interarm region, 3) Perseus arm, 4) beyond the Perseus arm.

${ }^{\mathrm{c}}$ Some of parameters are consistent with 0 and thus are not well determined. We present them for reference. 
TABLE 3

NUMBER OF COUNTS IN EACH ENERGY BIN.

\begin{tabular}{cc}
\hline \hline Energy $(\mathrm{GeV})$ & Counts \\
\hline $0.10-0.14$ & 26673 \\
$0.14-0.20$ & 71637 \\
$0.20-0.28$ & 91336 \\
$0.28-0.40$ & 93286 \\
$0.40-0.56$ & 78330 \\
$0.56-0.80$ & 61337 \\
$0.80-1.13$ & 45386 \\
$1.13-1.60$ & 30713 \\
$1.60-2.26$ & 19351 \\
$2.26-3.20$ & 11301 \\
$3.20-4.53$ & 6426 \\
$4.53-6.40$ & 3761 \\
$6.40-9.05$ & 2095 \\
$9.05-25.6$ & 2333 \\
\hline
\end{tabular}

TABLE 4

LOG-LIKELIHOOd AND EMISSIVITIES FOR SEVERAL CHOICES OF $T_{\mathrm{S}}$.

\begin{tabular}{ccccc}
\hline \hline$T_{\mathrm{S}}$ & $\ln (L)$ & $q_{\mathrm{HI}, 1}(E \geq 100 \mathrm{MeV})$ & $q_{\mathrm{HI}, 2}(E \geq 100 \mathrm{MeV})$ & $q_{\mathrm{HI}, 3}(E \geq 100 \mathrm{MeV})$ \\
\hline $100 \mathrm{~K}$ & 114407.6 & $1.32 \pm 0.04$ & $1.27 \pm 0.05$ & $0.86 \pm 0.06$ \\
$125 \mathrm{~K}$ & 114480.1 & $1.47 \pm 0.05$ & $1.26 \pm 0.06$ & $1.14 \pm 0.08$ \\
$250 \mathrm{~K}$ & 114533.8 & $1.62 \pm 0.04$ & $1.35 \pm 0.05$ & $1.53 \pm 0.06$ \\
$400 \mathrm{~K}$ & 114544.5 & $1.67 \pm 0.07$ & $1.39 \pm 0.08$ & $1.64 \pm 0.09$ \\
optically-thin & 114552.8 & $1.70 \pm 0.07$ & $1.39 \pm 0.07$ & $1.77 \pm 0.09$ \\
\hline
\end{tabular}

${ }^{\mathrm{a}}$ Units: $q_{\mathrm{HI}, \mathrm{i}}\left(10^{-26}\right.$ photons $\mathrm{s}^{-1} \mathrm{sr}^{-1} \mathrm{H}$-atom $\left.{ }^{-1}\right)$

${ }^{\mathrm{b}}$ The subscripts refer to four regions defined to perform the analysis; 1) Local arm, 2) interarm region, 3) Perseus arm. 